\title{
Postbreakthrough behavior in flow through porous media
}

\author{
Eduardo López, ${ }^{1}$ Sergey V. Buldyrev, ${ }^{1}$ Nikolay V. Dokholyan, ${ }^{1,2,3}$ Leo Goldmakher, ${ }^{1}$ Shlomo Havlin, ${ }^{1,4}$ Peter R. King, ${ }^{5}$ \\ and H. Eugene Stanley ${ }^{1}$ \\ ${ }^{1}$ Center for Polymer Studies, Department of Physics, Boston University, Boston, Massachusetts 02215 \\ ${ }^{2}$ Department of Chemistry and Chemical Biology, Harvard University, 12 Oxford Street, Cambridge, Massachusetts 02138 \\ ${ }^{3}$ Department of Biochemistry and Biophysics, University of North Carolina at Chapel Hill, School of Medicine, \\ Chapel Hill, North Carolina 27599 \\ ${ }^{4}$ Minerva Center and Department of Physics, Bar-Ilan University, Ramat Gan, Israel \\ ${ }^{5}$ Center for Petroleum Studies, TH Huxley School, Imperial College, Prince Consort Road, London SW7 2BP, United Kingdom
}

(Received 16 December 2002; published 28 May 2003)

\begin{abstract}
We numerically simulate the traveling time of a tracer in convective flow between two points (injection and extraction) separated by a distance $r$ in a model of porous media, $d=2$ percolation. We calculate and analyze the traveling time probability density function for two values of the fraction of connecting bonds $p$ : the homogeneous case $p=1$ and the inhomogeneous critical threshold case $p=p_{c}$. We analyze both constant current and constant pressure conditions at $p=p_{c}$. The homogeneous $p=1$ case serves as a comparison base for the more complicated $p=p_{c}$ situation. We find several regions in the probability density of the traveling times for the homogeneous case $(p=1)$ and also for the critical case $\left(p=p_{c}\right)$ for both constant pressure and constant current conditions. For constant pressure, the first region $\mathrm{I}_{P}$ corresponds to the short times before the flow breakthrough occurs, when the probability distribution is strictly zero. The second region $\mathrm{II}_{P}$ corresponds to numerous fast flow lines reaching the extraction point, with the probability distribution reaching its maximum. The third region $\mathrm{III}_{P}$ corresponds to intermediate times and is characterized by a power-law decay. The fourth region $\mathrm{IV}_{P}$ corresponds to very long traveling times, and is characterized by a different power-law decaying tail. The power-law characterizing region $\mathrm{IV}_{P}$ is related to the multifractal properties of flow in percolation, and an expression for its dependence on the system size $L$ is presented. The constant current behavior is different from the constant pressure behavior, and can be related analytically to the constant pressure case. We present theoretical arguments for the values of the exponents characterizing each region and crossover times. Our results are summarized in two scaling assumptions for the traveling time probability density; one for constant pressure and one for constant current. We also present the production curve associated with the probability of traveling times, which is of interest to oil recovery.
\end{abstract}

DOI: 10.1103/PhysRevE.67.056314

PACS number(s): 47.55.Mh, 05.60.Cd, 64.60.Ak

\section{INTRODUCTION}

The problem of oil extraction from a reservoir and how this extraction evolves in time, is of significance to oil companies. In order to maximize profits, they must be able to predict how much oil is recoverable from a reservoir over a given period of time. This problem can be approached scientifically by devising a model that accurately predicts the behavior of oil during the extraction process.

Most oil reservoirs are complicated geological structures [1] composed of several kinds of rock that have been deposited over a long period of time. The configuration of the structure has usually been altered by tectonic activity and mineral deposition by aquifer flow. For our purposes, the types of rock comprising any oil reservoir can be separated into two categories: high permeability (conducting) and lowpermeability (insulating). The location of both the conducting and insulating rocks is random but also nontrivial, i.e., during extraction it is only through the conducting rock that the flow of oil occurs.

Based on the random spatial location of the conducting rock, a simplifying procedure that has emerged in attempting to predict oil extraction is to model the reservoir by a bond percolation cluster with occupation probability $p[2-20]$. The value of $p$ corresponds to the fraction of conducting rock to total rock of the reservoir. This captures the essential features of the reservoir, while avoiding some of the noncrucial complications. An additional advantage to this approach is that it makes available to the analysis of oil recovery the wealth of information pertaining to percolation theory. However, we must caution the reader that these approximations cannot be taken as the definitive description for oil reservoirs. It is well known [21-25] that true field-size porous media possess correlations. Our description merely represents a first-order approximation, which can serve as a base for more comprehensive studies.

To fully explain the flow of oil in a percolation cluster, several physical and geometric parameters of percolation have been explored. Among them, there is work on the shortest path in a percolation cluster $[9,11,13,26-33]$, the average flow time of a fluid inside a cluster [34], and the full probability distribution of flow time inside a cluster for relatively short times [12]. The multifractal nature of flow inside a cluster has also been the subject of a number of papers $[35,36]$.

In this work, we focus on one particular technique of oil extraction used by companies: secondary oil extraction. It consists in injecting a fluid (water, carbon dioxide or methane) into the reservoir in order to displace the oil trapped inside. The fluid is injected through an injection well, located 
at point $A$ of the reservoir, and the mixture of fluid and oil is collected at point $B$ of the reservoir, where an extraction well is placed. The distance between the wells is known as the interwell space $r$. Inside the reservoir medium, a mixture of two fluids is formed, driven by a pressure difference between wells $A$ and $B$. Here, we consider the case when the two fluids have equal viscosities and are both incompressible, and thus can be considered as identical miscible fluids. For fluids of different viscosities, see Refs. [37-41]. To predict the amount of oil obtained at a given time, one must understand the evolution of this flow. In particular, it is important to know how long the injected fluid starting at $A$ requires to reach $B$. Since the flow on the reservoir occurs in the set of paths that connect $A$ and $B$, knowledge of the traveling time $t$ (also known in the literature as first-passage time) on all the paths in the reservoir determines how much of the displacing fluid has reached $B$ at a given time and, therefore, what percentage of the extracted mixture still corresponds to oil.

Some progress has been made in the study of this problem. The case of homogeneous flow (with $p=1$ ), when the reservoir is only composed of conducting rock, has been treated in Ref. [15]. Also, the convective and diffusive regimes of a more realistic nonhomogeneous reservoir (specifically at criticality $p=p_{c}$ ) have been considered $[12,13,15]$. The time it takes for any amount of the injected fluid to reach $B$, called the breakthrough time $t_{\mathrm{br}}$, was analyzed in Refs. $[12,13]$. The case of different viscosities for the injected and displaced fluids has been studied before, both for a finite value of the viscosity ratio [39-41], and in the limit of very high ratio $[37,38]$. In the latter case, a behavior analogous to diffusion-limited aggregation in percolation is found.

In this paper, we analyze analytically and numerically the flow inside a two-dimensional (2D) bond percolation cluster for two different occupation probabilities $(p=1$ and $p$ $=p_{c}$ ) and under two different pumping conditions at $A$ : constant current and constant pressure. The quantity measured is the probability distribution of the traveling time $t$ of tracer particles after breakthrough in a percolation ensemble, for a given interwell space $r$, and a reservoir of linear dimension $L$. These measurements ultimately allow us to write the probability distributions in concise expressions, valid for all the conditions studied here.

In Sec. II, we introduce the basic mathematical quantities, the probability density of traveling times and the production curve, and relate them to the physical picture. Section III deals with the homogeneous case $p=1$, which serves as a template to understand the more complicated $0<p<1$ case. Section IV introduces the inhomogeneous model at criticality to be used, while Sec. V recounts the numerical results of the model. In Sec. VI, we present analytical arguments explaining the behaviors observed in Sec. V and these arguments are then used to present the final forms for the probability distributions under both constant current and constant pressure conditions.

\section{PRELIMINARY CONCEPTS}

In secondary oil extraction, a fluid (typically water) is injected into the reservoir through an injection or pumping well $A$, pushing oil trapped inside toward the extraction well $B$. We call any infinitesimal fluid element of water a tracer. The time it takes a tracer to travel from $A$ to $B$ is called the traveling time t. For each possible configuration of the reservoir, there are generally many possible paths to travel from $A$ to $B$ and each of these paths is called a streamline. Because of the multiplicity of streamlines, each particular tracer that starts at $A$, in general, requires a different time $t$ to reach $B$. The breakthrough time $t_{\mathrm{br}}$ corresponds to the time when the first water stream reaches $B$ for a given realization. We define $P(t, r, L) d t$ as the probability that a tracer crosses from $A$ to $B$ in a time between $t$ and $t+d t$, with the condition that $A$ and $B$ are separated by a distance $r$, in a reservoir of linear size $L$. The function $P(t, r, L)$ is averaged over all possible reservoir configurations connecting the wells. Physically, it represents the fraction of water that becomes part of the extracted mixture at time $t$. Note that when $t<t_{\mathrm{br}}, P(t, r, L)$ $=0$ and the mixture corresponds to oil only. For $t \geqslant t_{\mathrm{br}}$, certain tracers begin to reach $B$, and $P(t, r, L)>0$, giving a mixture of oil and water at $B$. As $t \rightarrow \infty, P(t, r, L) \rightarrow 0$, because no new streamlines reach $B$ and this well produces only water. Cost constraints dictate the point at which the use of the well is terminated.

From our knowledge of $P(t, r, L)$, assuming that the streamlines do not change over time, we can determine the average production curve $C(t, r, L)$, which is the ratio of oil contained in the mixture coming out of the extraction well at time $t$,

$$
C(t, r, L) \equiv 1-\int_{0}^{t} P(\tilde{t}, r, L) d \tilde{t} .
$$

Equation (1) exhibits the expected features of the mixture: initially $P(t, r, L)=0$ and only oil comes out, giving $C(t, r, L)=1$. As $t$ increases, $C(t, r, L)$ begins to decay as a function of the number of streamlines that reach the extraction well. As $t \rightarrow \infty$, all water streamlines reach the extraction well and $C(t, r, L)=0$.

For a reservoir being exploited with a pressure differential between $A$ and $B$, such that the total current between these two points is $Q$, the total amount of oil, $S$, contained inside the reservoir can be determined by the expression

$$
S=Q \int_{0}^{\infty} C(t) d t
$$

Assuming oil is incompressible and using units for which its density is equal to $1, S$ also represents the accessible volume of the reservoir. Inserting Eq. (1) into Eq. (2) and integrating by parts, we obtain

$$
\frac{S}{Q}=\int_{0}^{\infty} d t\left(1-\int_{0}^{t} P(\tilde{t}, r, L) d \tilde{t}\right)=\int_{0}^{\infty} t P(t, r, L) d t=\langle t\rangle .
$$

This result corresponds to the equal-time theorem, which states that the average traveling time of tracers inside the 
reservoir is equal to the available reservoir volume divided by the total external current $[15,42]$. Equation (A3) of the Appendix represents the same result, applied to a reservoir in which all the sand is conducting.

\section{HOMOGENEOUS CASE}

The homogeneous case $(p=1)$, which can be analytically solved, serves as a guide for the more realistic, nonanalytic critical percolation threshold case $\left(p=p_{c}\right)$. The $p=1$ case was studied in Ref. [43] using differential equations. Here, we obtain the solutions by conformal mapping, reproducing the results of Ref. [43], and add the particular form of the production curve for the times $t$ right after the breakthrough. This section contains the main results of the time behavior, but the derivations are located in the Appendix. One of the results obtained there is that $t$ carries units of $r^{2}$ and, therefore, in the following, $t$ is replaced by a scaled variable

$$
t^{\prime} \equiv \frac{t}{r^{2}}
$$

We assume that the reservoir is a circle of diameter $L$ centered at the origin and the two points $A$ and $B$ are located at $(-r / 2,0)$ and $(r / 2,0)$. We study the production curve $C(t, r, L)$. The ratio $L / r$ is represented by $\lambda$. Using $t^{\prime}$ and $\lambda$, the production curve $C(t, r, L)$ for a given $r$ and $L$ is

$$
C\left(t^{\prime}, \lambda\right) \equiv C\left(t / r^{2}, 1, \lambda\right)=C(t, r, L),
$$

where we have made use of Eq. (A12), which expresses the scaling rule of $C(t, r, L)$ under rescaling of $t$ to $t / r^{2}$. For the sake of brevity, we refer to $C\left(t^{\prime}, \lambda\right)$ as $C\left(t^{\prime}\right)$.

Originally, the reservoir is filled with one kind of fluid (oil). At time $t^{\prime}=0$, we start to inject at point $A$, with constant rate, a different fluid (water). We measure the production curve $C\left(t^{\prime}\right)$ at well $B$ (Fig. 1). We assume that both fluids are incompressible and have zero viscosity. The flow is then described by Darcy's Law $\vec{v}=-\kappa \vec{\nabla} P$ and $\nabla^{2} P=0$. A set of regions, and two transition times $t_{1 / 2}^{\prime}$ and $t_{\ell}^{\prime}$ separating these regions, appears for the production curve $C\left(t^{\prime}\right)$. We now present them.

(1) Region $I_{H^{*}}$. Before $t^{\prime}=t_{\mathrm{br}}^{\prime}(\lambda)$, the concentration of injected fluid at point $B$ is zero and only oil exits through point $B$. We denote this time region, when $t^{\prime} \leqslant t_{\mathrm{br}}^{\prime}$, as region $\mathrm{I}_{H}$.

(2) Region $I I_{H}$. When the injected fluid reaches the production well, the concentration of the displaced fluid rapidly drops immediately after breakthrough as

$$
C\left(t^{\prime}\right) \sim 1-K(\lambda) \sqrt{t^{\prime}-t_{\mathrm{br}}^{\prime}}
$$

This behavior occurs until $t^{\prime}=t_{1 / 2}^{\prime}(\lambda)$, when a new time dependence sets in. The time region $t_{\mathrm{br}}^{\prime}<t^{\prime} \leqslant t_{1 / 2}^{\prime}$ is defined as region $\mathrm{II}_{H}$.

(3) Region $I I I_{H}$. If $\lambda \gg 1$, for times $t^{\prime}>t_{1 / 2}^{\prime}$ there exists a region of power-law decay [43],

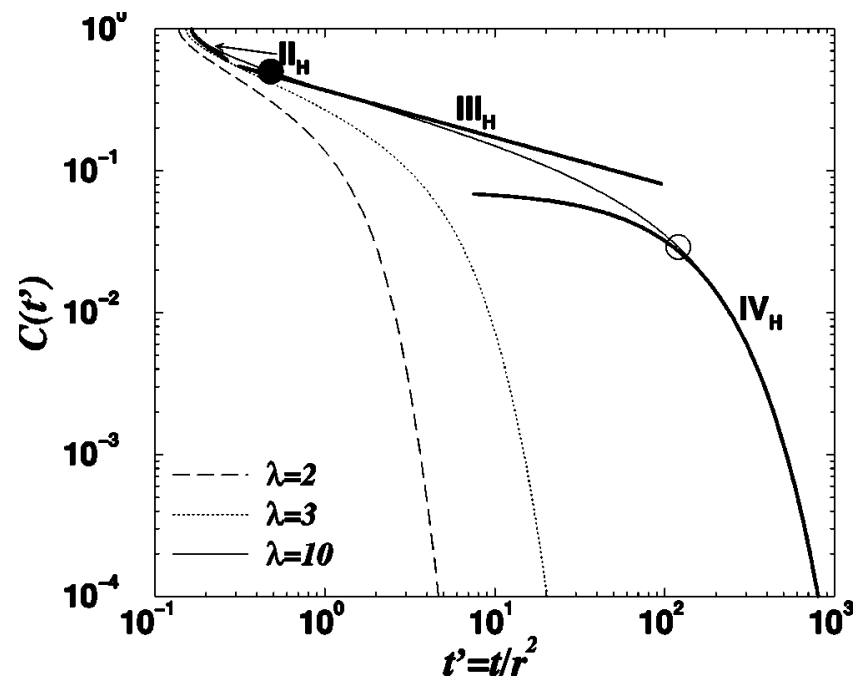

FIG. 1. Production curves for the homogeneous $p=1$ case in logarithmic scale for $\lambda=2,3$, and 10 . The solid curves superimposed to the production curves indicate the asymptotic behaviors of regions $\mathrm{II}_{H}, \mathrm{III}_{H}$, and $\mathrm{IV}_{H}$. All the solid lines have been calculated using the results in the Appendix. For region $\mathrm{II}_{H}$, Eq. (A10) is used with $K$ given by Eq. (A15); for region $\mathrm{III}_{H}$, Eq. (A11) is used, and for region $\mathrm{IV}_{H}$, we applied Eq. (A20). The full circle indicates the transition time $t_{1 / 2}^{\prime}$ and the empty circle indicates $t_{\ell}^{\prime}$.

$$
C\left(t^{\prime}\right) \sim t^{\prime-1 / 3}
$$

We call this region $\mathrm{III}_{H}$. It is present at times $t_{1 / 2}^{\prime}<t^{\prime} \leqslant t_{\ell}^{\prime}$, where $t_{\ell}^{\prime}(\lambda)$ is defined below.

(4) Region $I V_{H}$. At the transition time $t_{\ell}^{\prime}(\lambda)$, the reservoir is almost exhausted of oil and $C\left(t^{\prime}\right)$ decays exponentially as

$$
C\left(t^{\prime}\right) \sim \exp \left(-\left(t^{\prime}-t_{o}^{\prime}\right) / t_{\ell}^{\prime}\right)
$$

where $t_{o}^{\prime}(\lambda)$ is given by Eq. (A19). This is region $\mathrm{IV}_{H}$ and it is present in $C\left(t^{\prime}\right)$ for all $\lambda>1$, for times $t^{\prime}>t_{\ell}^{\prime}$. For $\lambda$ $=1, C\left(t^{\prime}, 1\right)=2 C\left(2 t^{\prime}, \infty\right)-1$ and the reservoir is completely exhausted at $(1 / 2) t_{1 / 2}^{\prime}(\infty)$.

(5) Transition times $t_{1 / 2}^{\prime}$ and $t_{\ell}^{\prime}$. Regions $\mathrm{II}_{H}$ and $\mathrm{III}_{H}$ are separated by a transition or crossover time $t_{1 / 2}^{\prime}(\lambda)$, given by Eq. (A16). Regions $\mathrm{III}_{H}$ and $\mathrm{IV}_{H}$ are separated by the crossover time $t_{\ell}^{\prime}(\lambda)$, given by Eq. (A18). For $\lambda \sim 1$, the two crossover times become of the same order, and region $\mathrm{III}_{H}$ is no longer present. However, as $\lambda \rightarrow \infty$,

$$
t_{1 / 2}^{\prime}(\lambda) \rightarrow 1 / 2
$$

and [43]

$$
t_{\ell}^{\prime}(\lambda) \sim \lambda^{d_{l}} \quad\left(d_{l}=3\right) .
$$


That is, as $\lambda$ increases, region $\mathrm{III}_{H}$ appears and the transition time between $\mathrm{III}_{H}$ and $\mathrm{IV}_{H}$ scales as a cubic function of the geometric factor $\lambda$.

\section{MODEL FOR THE INHOMOGENEOUS CASE}

We represent the reservoir as a two-dimensional bond percolation cluster and choose points $(-r / 2,0)$ and $(r / 2,0)$, denoted by $A$ and $B$, respectively, to be the injection and extraction well positions. Points $A$ and $B$ are separated by a geometric distance $r$ and the system box has corners at $( \pm L / 2, \pm L / 2)$. We construct a percolation cluster by removing bonds of a square $L \times L$ lattice with probability $(1-p)$. Equivalently, each bond of the lattice is conducting with probability $p$ and insulating with probability $1-p$. We simulate the flow if the points $A$ and $B$ belong to the same conducting cluster. Otherwise, we discard the configuration. We restrict our simulations to the critical bond percolation probability $p=p_{c}=0.5$ [2]. We consider both spanning clusters and finite clusters, and perform averages over $10^{4}$ realizations of the medium.

To model the flow of water, we use the analogy with electrical circuits, where for each bond, the pressure drop corresponds to the voltage difference and the flow corresponds to the electrical current on the bond. A pressure difference between points $A$ and $B$ drives the tracer. We choose the value of the pressure difference according to the conditions desired: for constant pressure, we set a pressure difference of 1 between $A$ and $B$ for all realizations of the medium; for constant current, we first measure the resistance $R$ of the realization, and the pressure drop is chosen to be equal to $R$, so that the current for the realization is 1 . For each realization, $10^{4}$ tracers are introduced at point $A$ and then collected at point $B$. The set of all bonds with nonzero current contained in the percolation cluster for each particular realization defines the backbone of the cluster and the backbone mass $M_{B}$ is the number of bonds that constitute this backbone.

Mathematically, the "pressure" difference across bonds is equivalent to a "voltage" difference, so by solving Kirchoff's laws on the backbone, we obtain the potential (pressure) drops $\Delta P$ over all bonds for a given realization. For a node having $s$ outgoing bonds, the tracer selects each bond with a probability

$$
w_{i j}=\frac{\Delta P_{i j}}{\sum_{j} \Delta P_{i j}} \quad\left[j=1, \ldots, s \text { and } i=1, \ldots, M_{B}\right] .
$$

Here index $i$ is over the $M_{B}$ nodes and $j$ is over the $s$ outgoing bonds, i.e., the bonds for which the pressure at the node $i$ is larger than the pressure at the other node of the bond. For incoming bonds, $w_{i j}=0$. The time necessary to cross each bond is $t_{i j}=1 / \Delta P_{i j}$ and the velocity is $v_{i j}=\Delta P_{i j}$ since each bond has unit length. The total traveling time of a tracer is the sum of the times corresponding to all the bonds of the path connecting $A$ and $B$, chosen by this tracer. Since the
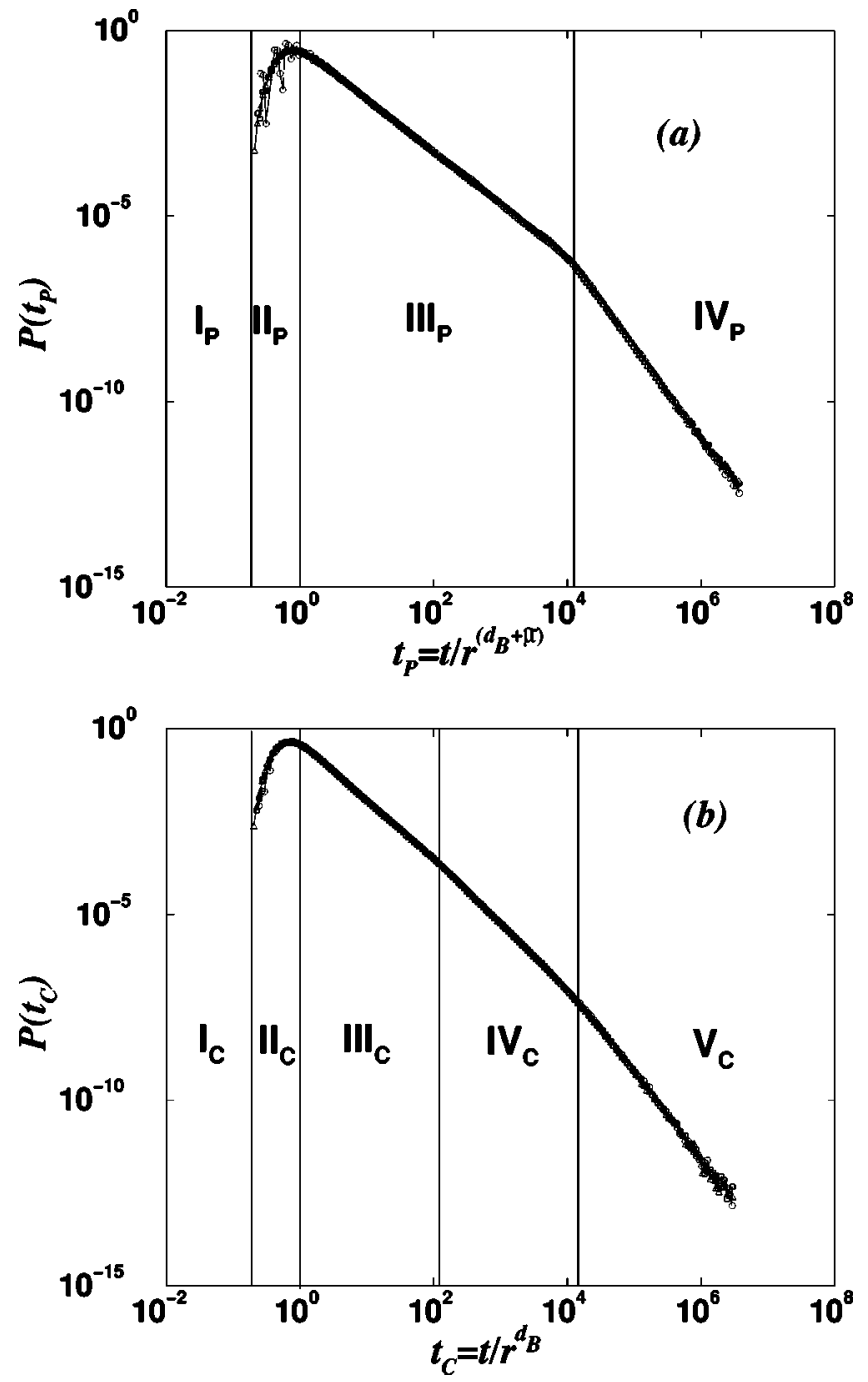

FIG. 2. (a) Probability distribution of traveling times for constant pressure conditions for $\lambda=32$. Three simulations are presented, for $L=258,514,1026$. (b) Probability distribution of traveling times for constant current conditions for $\lambda=32$. Three simulations are presented, for sizes $L=258,514,1026$.

particles do not interact with each other, it is equivalent to launching one particle at a time into the cluster. This procedure is known as particle launching algorithm $[15,44]$.

We determine the probability distribution of the traveling times $P(t, r, L)$ by counting the number of particles that travel from point $A$ to point $B$, separated by a distance $r$ in a box of linear size $L$, in a time between $t$ and $t+d t$, over all the particles and all realizations of the medium simulated.

\section{RESULTS FOR THE INHOMOGENEOUS CASE}

Following earlier work [13], and in a similar way to Sec. III, we define scaled times $t_{P}$ and $t_{C}$ to study the flow at both constant pressure and constant current conditions. For constant pressure, the scaled time is $t_{P} \equiv t / r^{d_{B}+\tilde{\mu}}$ and for constant current it is $t_{C} \equiv t / r^{d_{B}}$, where $d_{B}$ is the backbone fractal dimension and $\tilde{\mu}$ is the characteristic exponent of the resistivity dependence on distance. The current values for these 
TABLE I. Theoretical exponents and exponents obtained by simulation for all the regions and crossovers for functions $P\left(t_{C}\right)$ and $P\left(t_{P}\right)$. Exponents $\gamma^{(1)}, \gamma^{(2)}, d_{R}$, and $d^{\times}$are defined in Sec. VI and their numerical values along with their proposed theoretical values can be found in Table II. The value of exponent $\gamma^{(2)}$ was obtained as the average (avg.) over three simulations, having $L=258,514,1026$.

\begin{tabular}{|c|c|c|}
\hline \multicolumn{3}{|c|}{ Constant current } \\
\hline $\mathrm{III}_{C}$ & $\begin{aligned} g_{C}^{(1)} & =\min \left\{\left(d_{R}+g_{R}-2\right) /\left(d_{R}-1\right), \gamma^{(1)}\right\} \\
& =\min \{1.70 \pm 0.13,1.74 \pm 0.15\}\end{aligned}$ & $1.56 \pm 0.01$ \\
\hline $\operatorname{IV}_{C}(\lambda \approx 32)$ & $\begin{aligned} g_{C}^{(2)} & =\min \left\{\left(\gamma^{(1)}-1\right) d_{R}-g_{R}+2, \gamma^{(1)}\right\} \\
& =\min \{1.80 \pm 0.40,1.74 \pm 0.15\}\end{aligned}$ & $1.75 \pm 0.01$ \\
\hline $\operatorname{IV}_{C}(\lambda \rightarrow \infty)$ & $g_{C}^{(2)} \rightarrow g_{C}^{(1)} \approx 1.56 \pm 0.01$ & N/A \\
\hline $\mathrm{V}_{C}$ & $g_{C}^{(3)}=\gamma^{(2)}=2+a / \log _{10} L$ & $2.45 \pm 0.04$ (avg.) \\
\hline Crossover $\tau_{C}^{(1)}$ & $d_{C}^{(1)}=d_{\times}\left(d_{R}-1\right) / d_{R}=1.73 \pm 0.07$ & $1.74 \pm 0.05$ \\
\hline Crossover $\tau_{C}^{(2)}$ & $d_{C}^{(2)}=d_{\times}=2.84 \pm 0.10$ & $2.90 \pm 0.09$ \\
\hline \multicolumn{3}{|c|}{ Constant pressure } \\
\hline $\mathrm{III}_{P}$ & $g_{P}^{(1)}=\left(d_{R}+g_{R}-1\right) / d_{R}=1.43 \pm 0.08$ & $1.41 \pm 0.01$ \\
\hline $\mathrm{IV}_{P}$ & $g_{P}^{(2)}=\gamma^{(2)}=2+a / \log _{10} L$ & $2.43 \pm 0.06$ (avg.) \\
\hline Crossover $\tau_{P}$ & $d_{P}=d_{\times}=2.84 \pm 0.10$ & $2.78 \pm 0.15$ \\
\hline
\end{tabular}

exponents are $d_{B}=1.6432 \pm 0.0008$ and $\tilde{\mu}=0.9826 \pm 0.0008$ $[34,45]$. Under these new variables, we can define, in analogy with the homogeneous case [Eqs. (5) and (A12)], two new probability distributions:

$$
P\left(t_{C}, \lambda\right) \equiv P\left(t_{C}=t / r^{d_{B}}, 1, \lambda\right)
$$

for constant current conditions and

$$
P\left(t_{P}, \lambda\right) \equiv P\left(t_{P}=t / r^{d_{B}+\tilde{\mu}}, 1, \lambda\right)
$$

for constant pressure. Functions $P\left(t_{C}, \lambda\right)$ and $P\left(t_{P}, \lambda\right)$ are independent of $r$ and $L$, as reflected by the notation, but depend only on the ratio $\lambda$. Below, we refer to $P\left(t_{P}, \lambda\right)$ as $P\left(t_{P}\right)$ and to $P\left(t_{C}, \lambda\right)$ as $P\left(t_{C}\right)$, with the understanding that these functions are still dependent on $\lambda$. The two log-log plots, Figs. 2(a) and 2(b), contain three simulations each, corresponding to the probability distributions $P\left(t_{P}\right)$ and $P\left(t_{C}\right)$, respectively; both plots were prepared using $\lambda=32$ and system sizes $L=258,514,1026$. The curves overlap in both the constant pressure and constant current cases, which imply the scaling properties

$$
P\left(t_{C}, \lambda\right)=r^{d_{B}} P(t, r, L)
$$

and

$$
P\left(t_{P}, \lambda\right)=r^{d_{B}+\tilde{\mu}} P(t, r, L) .
$$

A set of regions with different behaviors appears in the two distributions. For the constant pressure distribution $P\left(t_{P}\right)$, we encounter the following. (1) Region $\mathrm{I}_{P}$, which corresponds to times smaller than the breakthrough time, before water reaches the extraction well; (2) region $\mathrm{II}_{P}$, appearing when the first water streams reach $B$ and ending at the onset of a constant slope region, including the maximum of $P\left(t_{P}\right)$;
(3) region $\mathrm{III}_{P}$, characterized by a constant value of the slope, defined as $-g_{P}^{(1)}$, of value $-1.41 \pm 0.01$; and (4) region $\mathrm{IV}_{P}$, which corresponds to another constant slope, defined as $-g_{P}^{(2)}$ and measured to be $-2.43 \pm 0.06$. The scaled crossover time from region $\mathrm{III}_{P}$ to region $\mathrm{IV}_{P}$ is called $\tau_{P}$.

The results for the constant current distribution $P\left(t_{C}\right)$ are similar to those of constant pressure. However, there is a difference in that there is yet another constant slope region present. Consequently, we have (1) region $\mathrm{I}_{C}$, for times before breakthrough; (2) region $\mathrm{II}_{C}$, corresponding to times right after breakthrough and until the first constant slope region appears, including the maximum of $P\left(t_{C}\right)$; (3) region $\mathrm{III}_{C}$, corresponding to a first slope, denoted by $-g_{C}^{(1)}$, of value $-1.56 \pm 0.01$; (4) region $\mathrm{IV}_{C}$, for a second slope $-g_{C}^{(2)}$, with a value of $-1.75 \pm 0.01$ for $\lambda=32$, but with a heavy dependence with respect to $\lambda$; and (5) region $\mathrm{V}_{C}$, with a slope represented by $-g_{C}^{(3)}$, measured to be -2.45 \pm 0.04 . The scaled crossover time between $\mathrm{III}_{C}$ and $\mathrm{IV}_{C}$ is defined as $\tau_{C}^{(1)}$ and between $\mathrm{IV}_{C}$ and $\mathrm{V}_{C}$, as $\tau_{C}^{(2)}$. All these values are presented in Table I.

Next, we present simulations for the probability distributions of traveling times for both constant current and constant pressure and for different $\lambda$ values (Fig. 3). As before, the distributions have two constant slope decay regions for constant pressure and three for constant current. However, as $\lambda$ changes, the positions of these regions change as well. The detailed shapes of $P\left(t_{P}\right)$ and $P\left(t_{C}\right)$ can be determined by studying their successive slopes, shown for constant pressure conditions in Fig. 4(a), and for constant current in Fig. 4(b). It is worth noting that regions $\mathrm{III}_{C}$ and $\mathrm{IV}_{C}$ become shorter as $\lambda$ decreases, with region $\mathrm{III}_{C}$ disappearing for $\lambda \leqslant 4$, and region $\mathrm{IV}_{C}$ for $\lambda \leqslant 2$.

Another set of quantities studied are the times for which the slopes cross over between regions. These crossover times 

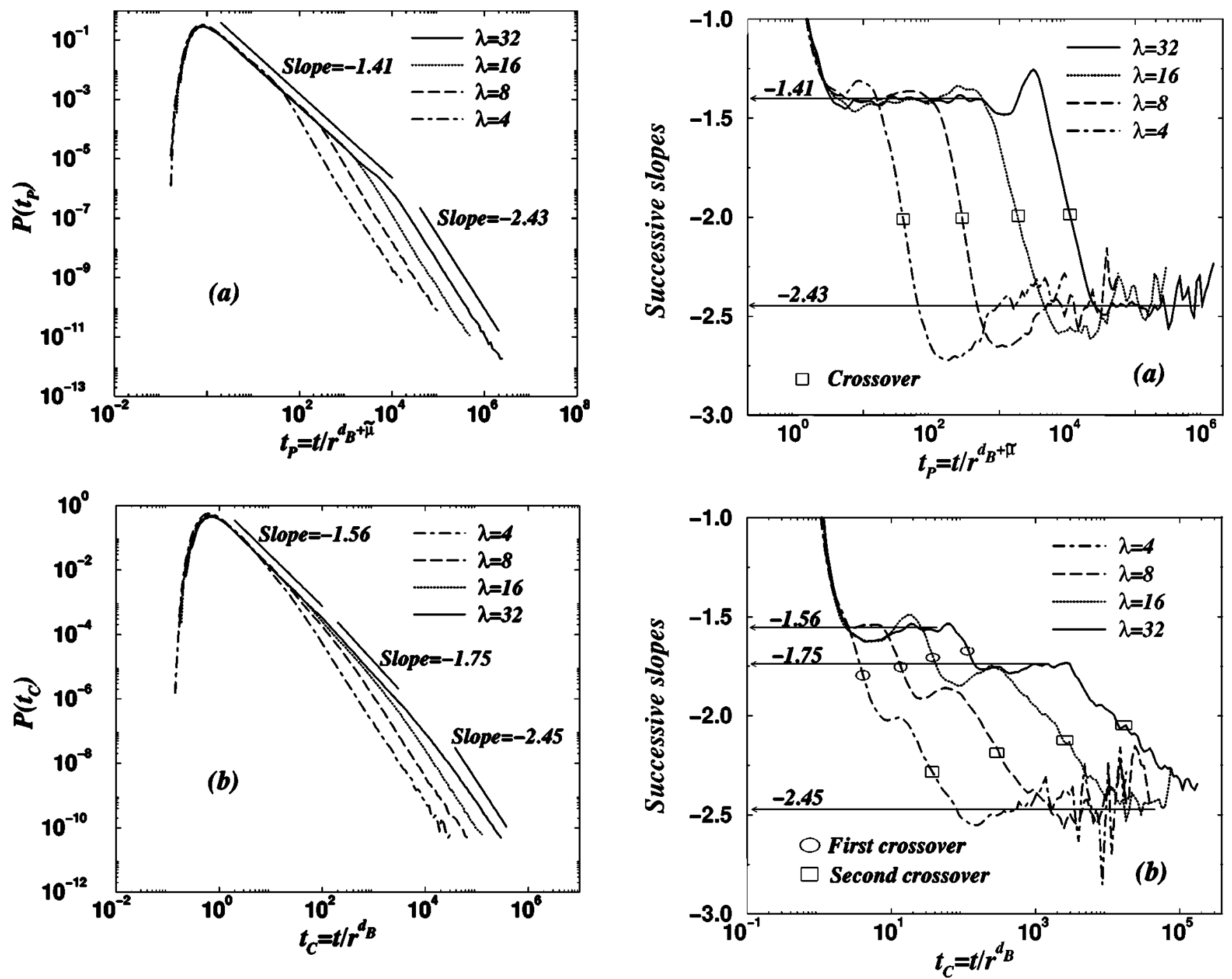

FIG. 3. (a) Probability distribution of traveling times for constant pressure conditions for system size $L=514$. Four simulations are presented, for $\lambda=4,8,16,32$. (b) Probability distribution of traveling times for constant current conditions for system size $L$ $=514$. Four simulations are presented, for $\lambda=4,8,16,32$.

are indicated by ellipses and boxes in Figs. 4(a) and 4(b). For the case of constant pressure, Fig. 5 shows the scaling of the crossover time $\tau_{P}$ with respect to $\lambda$, found to be $\tau_{P} \sim \lambda^{d_{P}}$ with $d_{P}=2.78 \pm 0.15$. For constant current, Figs. 6(a) and 6(b) show two crossover times $\tau_{C}^{(1)}$ and $\tau_{C}^{(2)}$, which scale as $\tau_{C}^{(1)} \sim \lambda^{d_{C}^{(1)}}$ and $\tau_{C}^{(2)} \sim \lambda^{d_{C}^{(2)}}$ with $d_{C}^{(1)}=1.74 \pm 0.05$ and $d_{C}^{(2)}$ $=2.90 \pm 0.09$, respectively. The positions of the crossover times have been determined by finding the positions of the inflection points of the successive slope plots.

Of practical interest is the production curve for constant current conditions (Fig. 7), because it supplies a tool for estimating the oil production efficiency. This curve is obtained by using Eq. (1), from the probability distribution of $P\left(t_{C}\right)$ [shown in Fig. 3(b)].

\section{DISCUSSION}

The distributions of traveling time under constant current and constant pressure conditions are connected to one an-

FIG. 4. (a) Successive slopes for the probability distribution of traveling times for constant pressure conditions for system size $L$ $=514$ (shown in Fig. 3). Four simulations are presented, for $\lambda=4,8,16,32$. (b) Successive slopes for the probability distribution of traveling times for constant current conditions for system size $L=514$. Four simulations are presented, for $\lambda=4,8,16,32$.

other. By definition, $t_{P}=\left(\widetilde{R} / r^{\tilde{\mu}}\right) t_{C}$, with $\widetilde{R}$ being the resistance of a percolation cluster [2,5], which is defined operationally as the voltage obtained between points $A$ and $B$ when a current of value 1 is present. The probability that a percolation cluster at the critical concentration $p_{c}$ has resistance $\widetilde{R}$ is given by $\widetilde{\Phi}(\widetilde{R}) d \widetilde{R}$, and can be seen in Fig. 8 . Approximately, $\widetilde{\Phi}(\widetilde{R})$ is given by

$$
\widetilde{\Phi}(\widetilde{R}) \sim \begin{cases}0, & \widetilde{R} \leqslant \widetilde{R}_{\text {min }} \\ \widetilde{R}^{-g_{R}}, & \widetilde{R}_{\text {min }}<\widetilde{R} \leqslant \widetilde{R}_{\text {max }} \quad\left[g_{R}=2.10 \pm 0.20\right] \\ 0, & \widetilde{R}>\widetilde{R}_{\text {max }},\end{cases}
$$




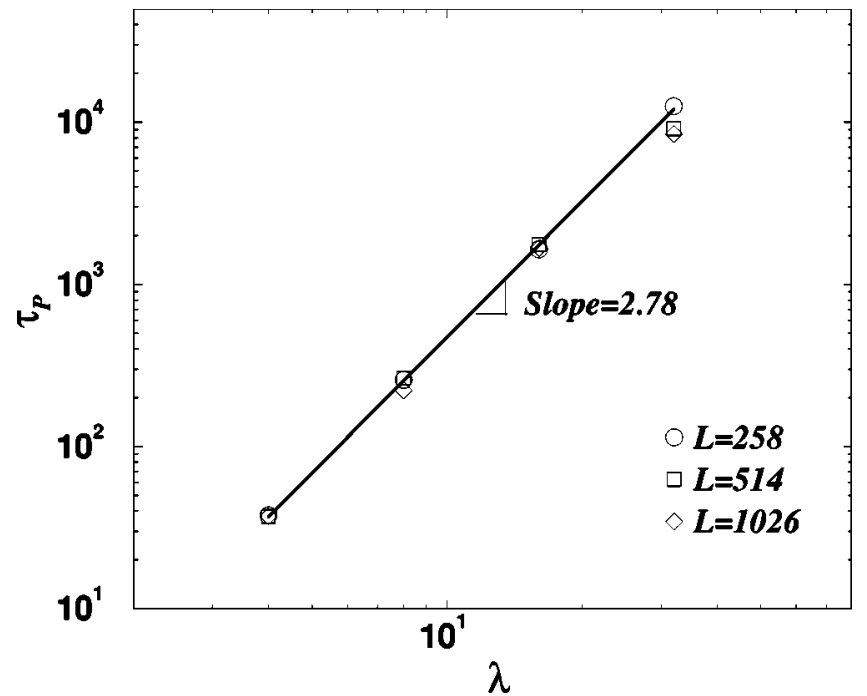

FIG. 5. Crossover times from region $\mathrm{III}_{P}$ to region $\mathrm{IV}_{P}$ for constant pressure conditions, with a system size of $L$ $=258,514,1026$ and $\lambda=4,8,16,32$.

with $\widetilde{R}_{\text {min }} \sim r^{\tilde{\mu}}$ and $\widetilde{R}_{\text {max }} \sim L^{\tilde{\mu}}$. The labels for $\widetilde{R}_{\text {min }}$ as minimum resistance, and for $\widetilde{R}_{\max }$ as maximum resistance, reflect the scaling rules of $\widetilde{R}$ with respect to the linear size $x$ of a cluster, i.e., $\widetilde{R} \sim x^{\tilde{\mu}}$ [2]. Therefore, since the interwell distance is $r, r^{\tilde{\mu}}$ represents the typical minimum value of resistance $\widetilde{R}_{\min }$. The maximum resistance is determined by the system size and it scales as $L^{\tilde{\mu}}$. Function $\widetilde{\Phi}(\widetilde{R})$ is not strictly zero for $\widetilde{R} \leqslant \widetilde{R}_{\text {min }}$ or $\widetilde{R}>\widetilde{R}_{\text {max }}$, but this is an acceptable approximation in our theory. All the results we present for $\Phi(\widetilde{R})$ are in agreement with previous work $[13,46]$.

As with the rescaled times, we find it convenient to define a scaled resistance $R=\widetilde{R} / r^{\tilde{\mu}}$ [13] and an associated probability $\Phi(R) \equiv r^{\tilde{\mu}} \widetilde{\Phi}\left(\widetilde{R}=R r^{\tilde{\mu}}\right)$. Function $\Phi(R)$ obeys the same scaling as $\widetilde{\Phi}(\widetilde{R})$ [it satisfies Eq. (16) dropping the tilde sign]. Quantity $R_{\text {min }} \equiv \widetilde{R}_{\text {min }} / r^{\tilde{\mu}}$ now becomes geometry independent and $R_{\max } \equiv \widetilde{R}_{\max } / r^{\tilde{\mu}}$ scales as $R_{\max } \sim \lambda^{\tilde{\mu}}$.

The redefined resistance $R$ reduces the relation between $t_{P}$ and $t_{C}$ to $t_{P}=R t_{C}$. Thus, $P\left(t_{P} \mid R\right)$ and $P\left(t_{C} \mid R\right)$ are related by $P\left(t_{C} \mid R\right) d t_{C}=P\left(t_{P} \mid R\right) d t_{P}$ and give

$$
P\left(t_{C} \mid R\right)=R P\left(t_{P} \mid R\right)
$$

Hence, the knowledge of one distribution enables us to calculate the other.

We focus on $P\left(t_{P}\right)$ initially. We consider parameter $R$, the scaled resistance of the cluster, as the relevant physical quantity dominating the properties of $P\left(t_{P}\right)$. Therefore, we numerically find $P\left(t_{P} \mid R\right) d t_{P}$, which corresponds to the probability that a tracer particle travels between points $A$ and $B$ in a cluster with resistance $R$ (more formally, with resistance within the range $R$ to $R+d R)$. Function $P\left(t_{P}\right)$ can be constructed by the convolution
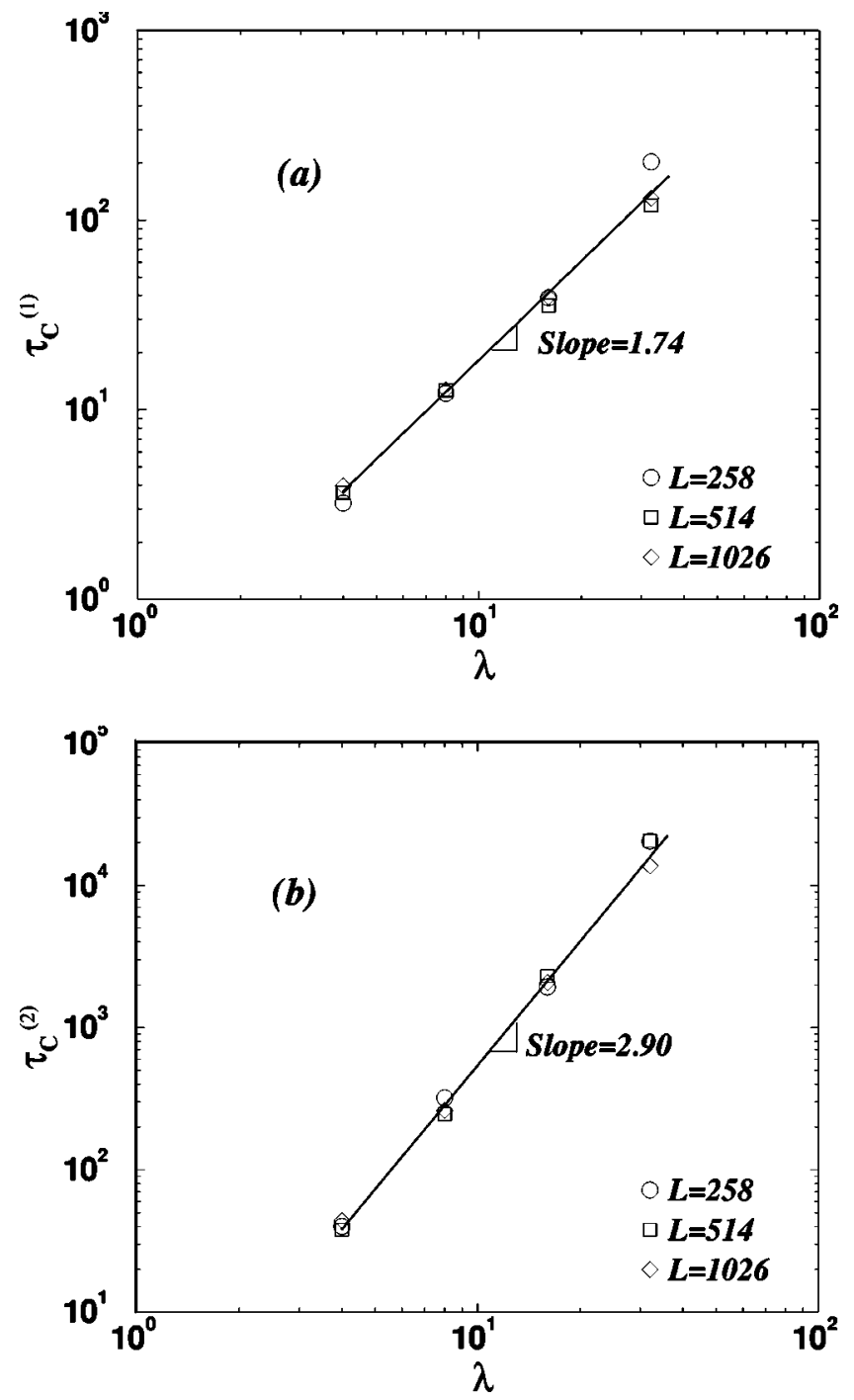

FIG. 6. (a) Crossover times from region $\mathrm{III}_{C}$ to region $\mathrm{IV}_{C}$ for constant current conditions, with system sizes $L$ $=258,514,1026$. (b) Crossover times from region $\mathrm{IV}_{C}$ to region $\mathrm{V}_{C}$ for constant current conditions, with system sizes $L$ $=258,514,1026$.

$$
P\left(t_{P}\right)=\int P\left(t_{P} \mid R\right) \Phi(R) d R
$$

Function $P\left(t_{P} \mid R\right)$, seen in Fig. 9, is obtained from the simulation of a system with $L=1026$ and $r=32$, or equivalently, with $\lambda=32$. Each curve represents a different value of the resistance $R$. The detailed behavior of the $P\left(t_{P} \mid R\right)$ has several features. The function reaches a maximum at time

$$
t_{P}^{*} \sim R^{d_{R}} \quad\left[d_{R}=2.57 \pm 0.02\right],
$$

as seen in Fig. 10. The scaled crossover time between the two power-law regions in Fig. 9, defined by the notation $t_{P}^{\times}$, occurs at equal time for all the curves, which indicates that it is independent of resistance $R$. However, we have performed other simulations with different values of $\lambda$, and have found that 


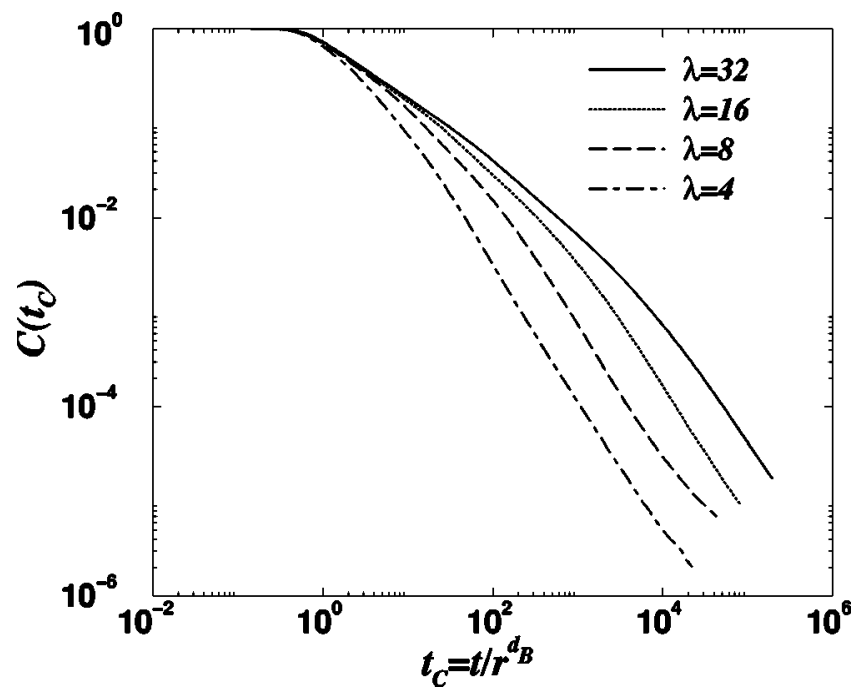

FIG. 7. Production curve for system size $L=1026$, under constant current conditions. Four interwell distances have been used, for $\lambda=4,8,16,32$.

$$
t_{P}^{\times} \sim \lambda^{d} \times \quad\left[d_{\times}=2.84 \pm 0.10\right]
$$

Region $t_{P}^{*} \leqslant t_{P}<t_{P}^{\times}$is characterized by a power-law decay of changing slope $-\gamma^{(1)}$ in the log-log plot (Fig. 9). For times $t_{P}$ close to $t_{P}^{*}$, the slope presents a value close to -2 , and as $t_{P} \rightarrow t_{P}^{\times}$, the value gradually changes to -1.6 (see Fig. 11). Additionally, as becomes apparent in Sec. VI E, $\gamma^{(1)}$ is also $\lambda$ dependent, a fact that affects the value of exponent $g_{C}^{(2)}$. We do not have a satisfactory explanation for this time dependence of $\gamma^{(1)}$, but as a first order approximation, in what follows, we assume it to be a constant, of value $\gamma^{(1)}=1.74$ \pm 0.15 , with the value given by its average over range $t_{P}^{*}$ $\leqslant t_{P}<t_{P}^{\times}$. The second region in Fig. 9, for times $t_{P} \geqslant t_{P}^{\times}$, is characterized by a slope of value $\gamma^{(2)}=2.41 \pm 0.04$. In analytical form, $P\left(t_{P} \mid R\right)$ can be approximated as

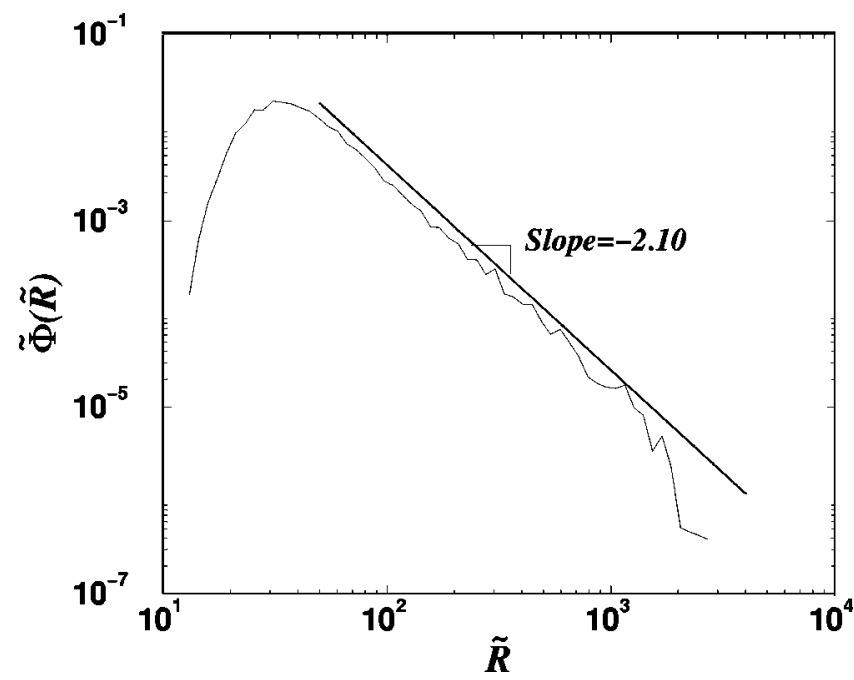

FIG. 8. Probability density $\widetilde{\Phi}(\widetilde{R})$ vs $\widetilde{R}$ for a simulation with system size $L=1026$ and $\lambda=32$.

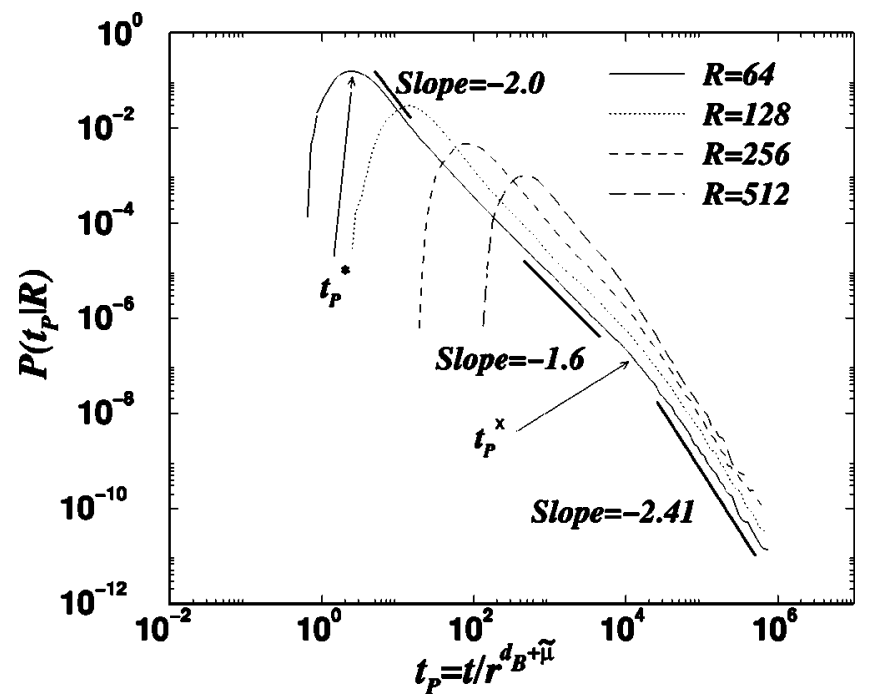

FIG. 9. Probability distribution $P\left(t_{P} \mid R\right)$ vs $t_{P}=t / r^{d_{B}+\tilde{\mu}}$ for system size $L=1026$ and interwell spacing $r=32(\lambda=32)$, under constant pressure conditions. The most probable traveling time $t_{P}^{*}$ and the crossover time $t_{P}^{\times}$are indicated for the curve corresponding to $R=64$.

$$
P\left(t_{P} \mid R\right) \sim\left\{\begin{array}{lll}
0, & t_{P}<t_{P}^{*} & \\
t_{P}^{-\gamma^{(1)}}, & t_{P}^{*}<t_{P}<t_{P}^{\times} & {\left[\gamma^{(1)}=1.74 \pm 0.15\right]} \\
t_{P}^{-\gamma^{(2)}}, & t_{P}>t_{P}^{\times} & {\left[\gamma^{(2)}=2.41 \pm 0.04\right]}
\end{array}\right.
$$

Taking $P\left(t_{P} \mid R\right)$ to be identical to zero for $t_{P}<t_{P}^{*}$, although an approximation, simplifies our calculations considerably and does not affect the validity of our results. In fact, this is a very good approximation since there are very few tracers reaching well $B$ in this time range, which means that their statistical contribution is negligible. This simplifying as-

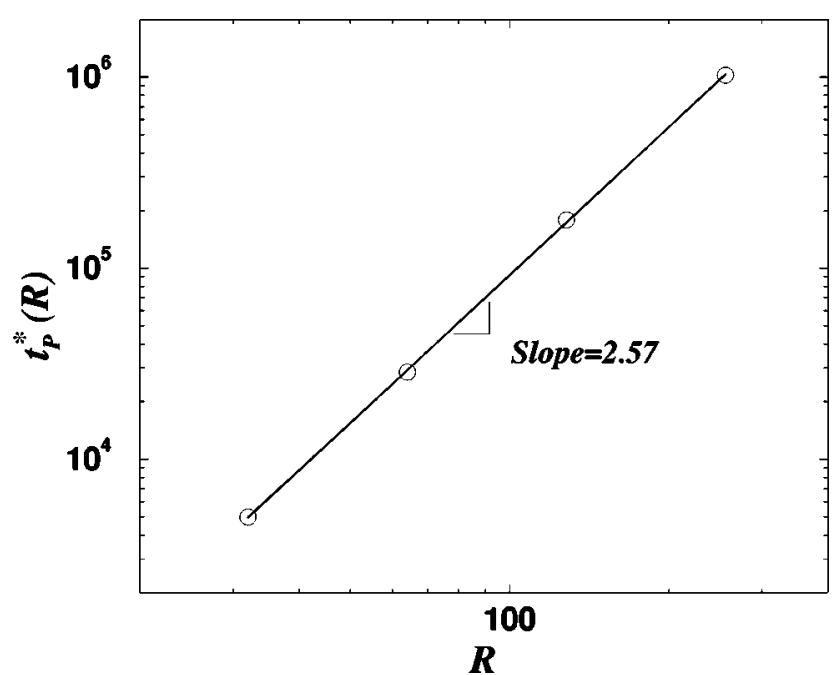

FIG. 10. Scaling of the maximum $t_{P}^{*}(R)$ of the probability $P\left(t_{P} \mid R\right)$ vs the resistance $R$, for a system size $L=1026$, and interwell distance $r=32(\lambda=32)$. The quantity $t_{P}^{*}(R)$ scales with $R$ as $R^{d_{R}}$, with $d_{R}=2.57 \pm 0.02$. 


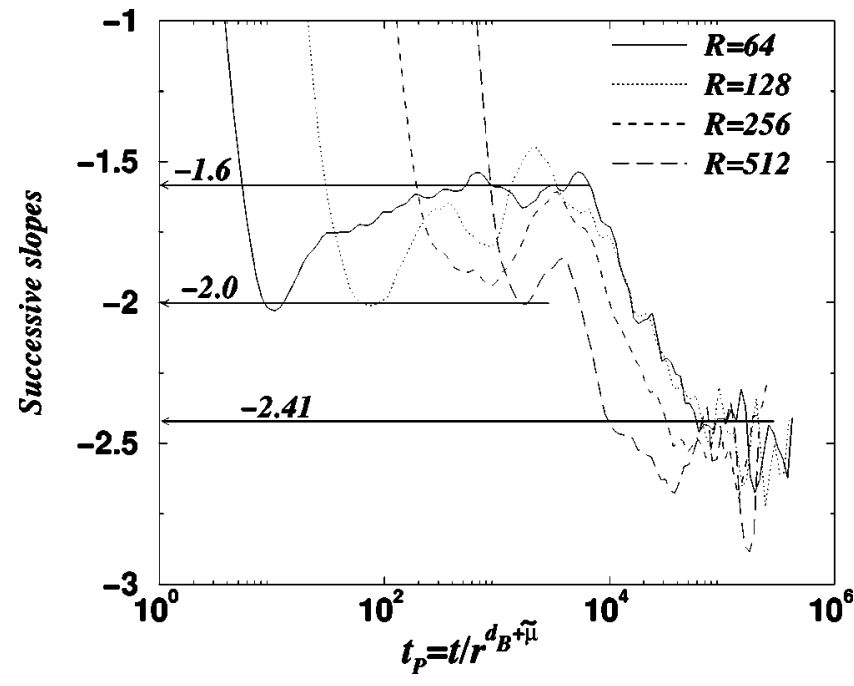

FIG. 11. Successive slopes for $P\left(t_{P} \mid R\right)$, with a system size $L$ $=1026$ and $\lambda=32$. The region corresponding to slope $\gamma^{(1)}$ of $P\left(t_{P} \mid R\right)$ varies in value, as indicated by the horizontal lines, between -1.60 and -2.00 . The second slope $\gamma^{(2)}$ reflects a more constant behavior, with value $2.41 \pm 0.04$.

sumption is repeated below for $P\left(t_{C} \mid R\right)$. The actual behavior of $P\left(t_{P} \mid R\right)$ for $t_{P}<t_{P}^{*}$ has been studied in detail before [13], where it was found to be that of a stretched exponential, carrying negligible statistical weight in our present calculations. In Sec. VI E, we incorporate this result into the full scaling ansatz for $P\left(t_{P}\right)$ and $P\left(t_{C}\right)$. The numerical values of the exponents of $P\left(t_{P} \mid R\right)$ and $\Phi(R)$ are summarized in Table II.

The similarity in the values of $d_{R}$ and the combination $1+d_{B} / \tilde{\mu}$ is worth some consideration. It has been proposed elsewhere [12] that under constant current conditions, the unscaled most probable traveling time scales with respect to $r$ as $r^{d_{B}}$. Now, for a fixed value of resistance $R$, at constant pressure conditions $\left(t_{P}=R t_{C}\right)$, we expect the unscaled most probable traveling time to scale as $r^{d_{B}+\tilde{\mu}}$, where scaling rule $\widetilde{R}=R r^{\tilde{\mu}}$ has been used. These considerations justify our
TABLE II. Exponents of functions $P\left(t_{P} \mid R\right)$ and $\Phi(R)$ determined through simulations, and also their proposed theoretical values. The symbol N/A represents not available. The value of the exponent $\gamma^{(2)}$ was obtained as the average (avg.) over three simulations, having $L=258,514,1026$.

\begin{tabular}{lcc}
\hline \hline Exponent & Theory & Simulation \\
\hline$\gamma^{(1)}$ & N/A & $1.74 \pm 0.15$ \\
$\gamma^{(2)}$ & $2+a / \log _{10} L$ & $2.41 \pm 0.04$ (avg.) \\
$d_{R}$ & $d_{B} / \tilde{\mu}+1=2.672 \pm 0.002$ & $2.57 \pm 0.02$ \\
$d_{\times}$ & $d_{B}+\tilde{\mu}=2.6258 \pm 0.0011$ & $2.84 \pm 0.10$ \\
$g_{R}$ & See Ref. [13] & $2.10 \pm 0.20$ \\
\hline \hline
\end{tabular}

choice of rescaled times. Since $\widetilde{R} \sim r^{\tilde{\mu}}$, we can assume that the most probable traveling time at constant $\widetilde{R}$ scales as $\widetilde{R}^{1+d_{B} / \tilde{\mu}}$ and thus, $d_{R}=d_{B} / \tilde{\mu}+1$. By similar arguments regarding the longest possible tracer trajectories inside the system, the unscaled crossover time can be related to $L^{d_{B}+\tilde{\mu}}$, indicating that $t_{P}^{\times} \sim \lambda^{d \times}$ would have $d_{\times}$be equivalent to $d_{B}$ $+\tilde{\mu}$. However, our numerical simulations do not have sufficient accuracy to answer this question definitively.

The properties just described for $P\left(t_{P} \mid R\right)$ and use of Eq. (18) determine the form of $P\left(t_{P}\right)$ as we now prove for each separate region identified in Figs. 2(a) and 2(b) (see also Sec. $\mathrm{V})$. The analytical expressions for all the predicted exponents pertaining to the distribution $P\left(t_{P}\right)$ and their numerical values are given in Table I.

\section{A. Region III $_{P}$}

To use $P\left(t_{P} \mid R\right)$ for the calculation of $P\left(t_{P}, \lambda\right)$, according to Eq. (18), we need to take into account its normalization factor, which is $R$ dependent. To obtain this factor, we integrate the distribution and equate it to unity, using the following assumptions: $P\left(t_{P} \mid R\right)$ is strictly equal to zero for times $t_{P}<t_{P}^{*} \sim R^{d_{R}}$ and, as a consequence of the previous condition, the lower limit of integration is $t_{P}^{*} \sim R^{d_{R}}$. The final form of $P\left(t_{P} \mid R\right)$ is

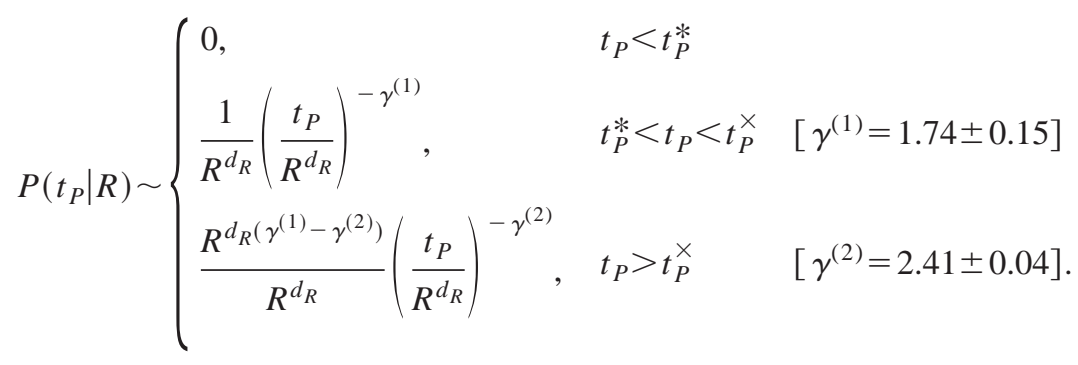

Regarding $\Phi(R)$, its normalization has no impact on the variables in which we are interested and is therefore not presented.

To perform the integration of Eq. (18), we must first define the limits of integration in $R$ that apply to region $\mathrm{III}_{P}$. The $R$ dependence of function $P\left(t_{P} \mid R\right)$ is contained in its normalization factor, and also in the fact that this distribution is nonzero when $t_{P}>t_{P}^{*} \sim R^{d_{R}}$. Therefore, for a given time 
$t_{P}$, the integrand of Eq. (18) is nonzero for all $R<t_{P}^{1 / d_{R}}$. The lower limit of the integral is $R_{\min }$, but it is irrelevant as regards the $t_{P}$ scaling form. For function $P\left(t_{P} \mid R\right)$, only the first scaling $t_{P}^{-\gamma^{(1)}}$ is used, since region $\mathrm{III}_{P}$ is limited to $t_{P}$ $\leqslant t_{P}^{\times}$. Then, using Eqs. (16), (22), and the convolution Eq. (18), we obtain

$$
P\left(t_{P}\right) \sim t_{P}^{-\gamma^{(1)}} \int{ }^{t / d_{R}} R^{-d_{R}\left(-\gamma^{(1)}+1\right)} R^{-g_{R}} d R
$$

for $t_{P} \leqslant t_{P}^{\times}$. After integration, the exponent of $t_{P}$ is positive, which implies that the leading term for the integral is the upper limit of integration. Substituting this limit yields the scaling form $t_{P}^{\left(1-d_{R}-g_{R}\right) / d_{R}}$. Therefore, since in region $\mathrm{III}_{P}$ the scaling is $P\left(t_{P}\right) \sim t_{P}^{-g_{P}^{(1)}}$, we obtain

$$
g_{P}^{(1)}=\frac{g_{R}+d_{R}-1}{d_{R}} .
$$

\section{B. Region $I V_{P}$ and crossover time $\tau_{P}$}

For times $t_{P}>t_{P}^{\times}$, distribution $P\left(t_{P} \mid R\right)$, regardless of the value of $R$, decays with a power-law of $t_{P}^{-\gamma^{(2)}}$. The upper limit of integration is determined by the largest resistance possible within the conditions of the geometry, which we denote by $R_{\max }$, and it is independent of $t_{P}$. Therefore, by convolution Eq. (18), we obtain

$$
P\left(t_{P}\right) \sim t_{P}^{-g_{P}^{(2)}}=t_{P}^{-\gamma^{(2)}}
$$

giving

$$
g_{P}^{(2)}=\gamma^{(2)}
$$

The actual numerical value of $g_{P}^{(2)}$ is discussed in Sec. VI D.

The crossover time $t_{P}^{\times}$separates regions $\mathrm{III}_{P}$ and $\mathrm{IV}_{P}$. Therefore, $\tau_{P}=t_{P}^{\times}$, which implies that

$$
\tau_{P} \sim \lambda^{d_{\times}}
$$

and

$$
d_{P}=d_{\times}
$$

\section{Mapping between $P\left(t_{P}\right)$ and $P\left(t_{C}\right)$}

Based on Eq. (17), the differences observed in the curves for $P\left(t_{P}\right)$ and $P\left(t_{C}\right)$ are caused by a shift of curve $P\left(t_{P} \mid R\right)$ on a $\log -\log$ plot by $-\log R$. Function $P\left(t_{C} \mid R\right)$, defined from $P\left(t_{P} \mid R\right)$ by Eq. (17), is characterized by two powerlaw decays with exponents $-\gamma^{(1)}$ and $-\gamma^{(2)}$, a maximum at time $t_{C}^{*} \equiv t_{P}^{*} / R \sim R^{d_{R}-1}$, and a scaled crossover time between the two power-law regions at $t_{C}^{\times} \equiv t_{P}^{\times} / R \sim R^{-1} t_{P}^{\times}$. In analytical form, $P\left(t_{C} \mid R\right)$ can be approximated by an equation similar to Eq. (22),

$$
P\left(t_{C} \mid R\right) \sim \begin{cases}0, & t_{C}<t_{C}^{*} \\ \frac{1}{R^{d_{R}-1}}\left(\frac{t_{C}}{R^{d_{R}-1}}\right)^{-\gamma^{(1)}}, & t_{C}^{*}<t_{C}<t_{C}^{\times} \quad\left[\gamma^{(1)}=1.74 \pm 0.15\right] \\ \frac{R^{d_{R}\left(\gamma^{(1)}-\gamma^{(2)}\right)}}{R^{d_{R}-1}}\left(\frac{t_{C}}{R^{d_{R}-1}}\right)^{-\gamma^{(2)}}, & t_{C}>t_{C}^{\times} \quad\left[\gamma^{(2)}=2.41 \pm 0.04\right],\end{cases}
$$

where the normalization factor has been obtained by assumptions analogous to those used for $P\left(t_{P} \mid R\right)$. To explain the different power-law regions in the behavior of $P\left(t_{C}\right)$, we use the convolution

$$
P\left(t_{C}\right)=\int P\left(t_{C} \mid R\right) \Phi(R) d R=\int P\left(t_{P} \mid R\right) R \Phi(R) d R,
$$

where Eq. (17) has been taken into account.

In Eq. (29), times $t_{C}^{*}$ and $t_{C}^{\times}$are dependent on $R$ and, therefore, the size of the scaling region $P\left(t_{C} \mid R\right) \sim t_{C}^{-\gamma^{(1)}}$ is $R$ dependent as well. This is an important difference between the constant current and constant pressure cases, because it is the cause of the existence of regions $\mathrm{III}_{C}$ and $\mathrm{IV}_{C}$ with exponents that differ from each other and from the exponent of region $\operatorname{III}_{P}$. The $R$ dependence of the size of the region is as follows: as $R$ increases, $t_{C}^{*}$ increases, but $t_{C}^{\times}$decreases. For large enough $R$, denoted here by $R_{\times}$, these two times coincide, and only the scaling $t_{C}^{-\gamma^{(2)}}$ is present. The resistance $R_{\times}$ scales as a function of the crossover time $t_{P}^{\times}$as $R_{\times}$ $\sim\left(t_{P}^{\times}\right)^{1 / d_{R}}$ and, ultimately, in terms of $\lambda$ as $R_{\times} \sim \lambda^{d_{\times} / d_{R}}$. Also, there is a specific time, denoted here by $M$, associated with this point of coincidence, where only the power-law decay with $t_{C}^{-\gamma^{(2)}}$ survives. Inserting $R_{\times}$into $t_{C}^{*} \sim R^{d_{R}-1}$ (or alternatively into $t_{C}^{\times} \sim R^{-1} t_{P}^{\times}$), we obtain

$$
M \sim \lambda^{\left(d_{R}-1\right) d_{\times} / d_{R}} .
$$

It is now possible to explain the existence of regions $\mathrm{III}_{C}$ and $\mathrm{IV}_{C}$. For times $t_{C}<M$, the convolution integral in Eq. (30) has an upper limit obtained by the relation $t_{C} \sim R^{d_{R}-1}$ and, consequently, the expression becomes 


$$
P\left(t_{C}\right) \sim t_{C}^{-\gamma^{(1)}} \int{ }^{t_{C} /\left(d_{R}-1\right)} R^{-\left(d_{R}-1\right)+\gamma^{(1)}\left(d_{R}-1\right)-g_{R}} d R .
$$

If the exponent of $t_{C}$ after integration is positive, only the upper limit is relevant and the integral yields $P\left(t_{C}\right)$ $\sim t_{C}^{\left(2-d_{R}-g_{R}\right) /\left(d_{R}-1\right)}$. Otherwise, the integral is finite and $P\left(t_{C}\right) \sim t_{C}^{-\gamma^{(1)}}$. For region $\mathrm{III}_{C}$, the scaling behavior is $P\left(t_{C}\right) \sim t_{C}^{-g_{C}^{(1)}}$, implying that

$$
g_{C}^{(1)}=\min \left\{\frac{d_{R}+g_{R}-2}{d_{R}-1}, \gamma^{(1)}\right\} .
$$

The predicted value for $g_{C}^{(1)}$ is different than the measured quantity, although it is within the error bars. This discrepancy is associated with the simplifications made in our theory, which do not reflect the fact that, for $t_{C}<M$, the highest contribution to $P\left(t_{C}\right)$ comes from lower values of $R$, and $\Phi(R)$ in this range decays with an exponent $g_{R}$ smaller than 2.1 [note the rounded shape of $\Phi(R)$ near its peak in Fig. 8]. Therefore, with a smaller effective value of $g_{R}$, the exponent $g_{C}^{(1)}$ acquires a smaller value as well.

As it follows from the behavior of $P\left(t_{P} \mid R\right)$, for times $t_{C}>M, P\left(t_{C} \mid R\right)$ scales as $t_{C}^{-\gamma^{(1)}}$ for small values of $R$ and as $t_{C}^{-\gamma^{(2)}}$ for larger $R$. The transition between the two situations occurs when $t_{C}=t_{C}^{\times}$, and since this last quantity scales as $R^{-1}, t_{C} \sim R^{-1}$ and the upper limit of the integral for the regime $t_{C}^{-\gamma^{(1)}}$ is $R \sim t_{C}^{-1}$. Therefore, the convolution Eq. (30) is written as

$$
\begin{aligned}
P\left(t_{C}\right) \sim & t_{C}^{-\gamma^{(1)}} \int{ }^{t_{C}^{-1}} R^{-\left(d_{R}-1\right)+\gamma^{(1)}\left(d_{R}-1\right)-g_{R}} d R \\
& +t_{C}^{-\gamma^{(2)}} \int_{t_{C}^{-1}}^{R_{\max }} R^{-\left(d_{R}-1\right)+\gamma^{(1)} d_{R}-\gamma^{(2)}-g_{R}} d R .
\end{aligned}
$$

Once again, by considering the value of the exponents after integration, given that $P\left(t_{C}\right) \sim t_{C}^{-g_{C}^{(2)}}$, we obtain for region $\mathrm{IV}_{C}$

$$
g_{C}^{(2)}=\min \left\{\left(\gamma^{(1)}-1\right) d_{R}-g_{R}+2, \gamma^{(1)}\right\} .
$$

This result is valid for a given value of $\lambda$ due to the dependence of $\gamma^{(1)}$ on this parameter. At the limit of very large $\lambda$, application of the equal-time theorem indicates that $g_{C}^{(2)}$ approaches $g_{C}^{(1)}$, or

$$
g_{C}^{(2)} \rightarrow g_{C}^{(1)} \quad[\lambda \rightarrow \infty],
$$

which corresponds to Eq. (59). See Sec. VI E for details.

Because $t_{C}^{\times} \sim R^{-1}$, as $R$ decreases, $t_{C}^{\times}$increases. Consequently, the transition of $P\left(t_{C} \mid R\right)$ to the scaling $P\left(t_{C} \mid R\right)$ $\sim t_{C}^{-\gamma^{(2)}}$ occurs at later times for smaller $R$. Since the minimum $R$ possible is $R_{\min }$, the crossover $t_{C}^{\times}$has an upper bound, denoted here by $N$. The form of $N$ is determined through $P\left(t_{C} \mid R=R_{\min }\right)$ and it is equal to $t_{C}^{\times}$for this particular distribution, or

$$
N \sim t_{C}^{\times} \sim R_{\min }^{-1} t_{P}^{\times} \sim \lambda^{d \times} .
$$

For $t_{C}>N$, we have $P\left(t_{C} \mid R\right) \sim t_{C}^{-\gamma^{(2)}}$. Using this form of $P\left(t_{C} \mid R\right)$ in the convolution Eq. (30) we obtain $P\left(t_{C}\right)$ $\sim t_{C}^{-\gamma^{(2)}}$. The upper limit of integration is again $R_{\max }$. Thus, for region $\mathrm{V}_{C}$, we have

$$
g_{C}^{(3)}=\gamma^{(2)} .
$$

This exponent is equal to that obtained for region $\operatorname{IV}_{P}$.

Now we derive the expression for the first crossover time $M$, which separates regions $\mathrm{III}_{C}$ and $\mathrm{IV}_{C}$. According to Eq. (31)

$$
\tau_{C}^{(1)}=M \sim \lambda^{d_{\times}\left(d_{R}-1\right) / d_{R}} .
$$

Comparing this with the definition for $\tau_{C}^{(1)}$ given in Sec. V, we obtain

$$
d_{C}^{(1)}=d_{\times}\left(d_{R}-1\right) / d_{R} .
$$

The second crossover, according to Eq. (37), occurs at $\tau_{C}^{(2)}=N$ and obeys the scaling

$$
\tau_{C}^{(2)} \sim \lambda^{d_{\times}},
$$

which, in turn, implies that

$$
d_{C}^{(2)}=d_{\times} .
$$

\section{Long-time regime for constant pressure and constant current}

As a first approximation, we can assume that functions $P\left(t_{P}\right)$ and $P\left(t_{C}\right)$, for a given ratio $\lambda$, have no dependence on the system size $L$. However, our numerical simulations suggest a weak dependence of exponents $g_{P}^{(2)}$ and $g_{C}^{(3)}$ on the system size $L$. These exponents express only the long-time behavior (large $t_{P}$ and $t_{C}$ or alternatively, regions $\mathrm{IV}_{P}$ and $\mathrm{V}_{C}$ ) of the distributions. The values of these exponents were found to be the same, since both $g_{P}^{(2)}$ and $g_{C}^{(3)}$ are equal to $\gamma^{(2)}$. Consequently, this means that $\gamma^{(2)}$ is a function of $L$. Based on the multifractal nature of flow in porous media [35] and, particularly, on the results obtained in Refs. [36,47], we propose an argument on how $\gamma^{(2)}$ depends on $L$. Since the scaling forms for $P\left(t_{C}\right)$ and $P\left(t_{P}\right)$ are the same at long times (regions $\mathrm{IV}_{P}$ and $\mathrm{V}_{C}$ ), for the rest of this section we introduce notation $\tilde{t}$ to represent both $t_{P}$ and $t_{C}$, because the following argument applies to both distributions.

Barthélémy et al. [36] studied the nature of the distribution of tracer velocities $\left\{v_{i}\right\}$ in a cluster connecting two points in percolation, and found that it has multifractal properties. Particularly, $\widetilde{P}\left(v_{i}\right) \sim v_{i}^{-1+a / \log _{10} L}$, where $v_{i}$ is the velocity of the tracer through bond $i$, and $a$ is a constant. What is the consequence of this distribution in terms of our prob- 


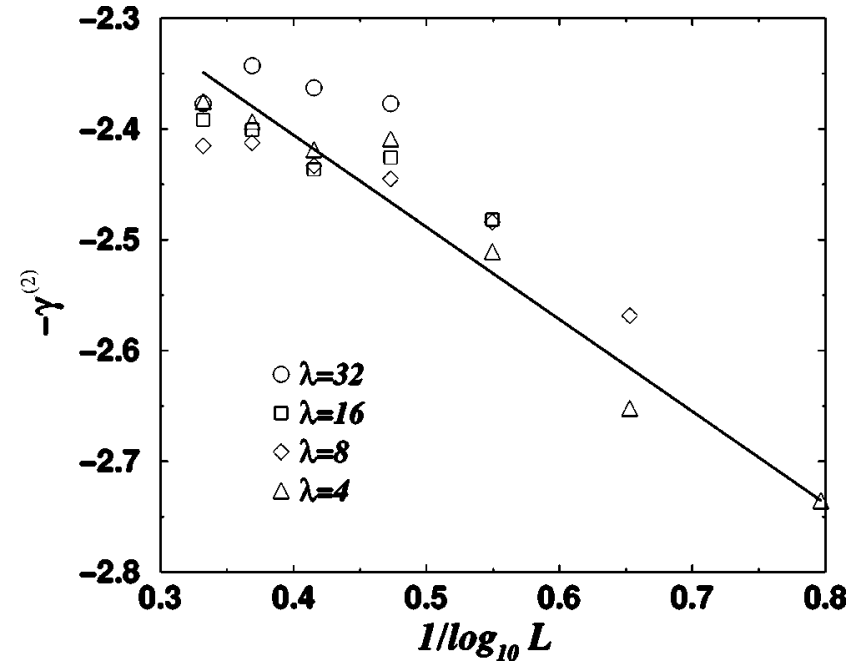

FIG. 12. Value of the tail exponent $-\gamma^{(2)}$ as a function of $1 / \log _{10} L$, where $L$ corresponds to the system size of the simulation. Each symbol represents the succession of values of $\gamma^{(2)}$ for a given ratio $\lambda$, with the longest curve corresponding to ratio 4 and the shortest to ratio 32 . The thick straight line represents the least squares fit of the $\lambda=4$ case, which yields a line of value $\gamma^{(2)}$ $=2.08+0.81 / \log _{10} L$, as stated in the text.

lem? From the rules of the simulation, we have $\widetilde{t}_{i}=1 / v_{i}$ and, therefore, the distributions of both time and velocity are related by $\widetilde{P}\left(v_{i}\right) d v_{i}=\widetilde{P}\left(\tilde{t}_{i}\right) d \tilde{t}_{i}$. If a tracer travels through a bond with a velocity $v_{i}$ that is among the lowest velocities on the realization, then it is true that $\tilde{t} \approx \tilde{t}_{i}$ i.e., the total time of the tracer is approximately equal to the time it takes to pass the slowest bond. Then $\widetilde{P}(\widetilde{t}) \approx \widetilde{P}\left(\tilde{t}_{i}\right)$ and

$$
\widetilde{P}(\widetilde{t}) \approx \widetilde{P}\left(\tilde{t}_{i}\right)=\widetilde{P}\left(v_{i}\right) \frac{d v_{i}}{d \tilde{t}_{i}} .
$$

Given that the tracers choose to travel through a particular bond with a probability proportional to the value of the velocity in that bond, we then have to modify the distribution $\widetilde{P}\left(v_{i}\right)$ for $v_{i} \widetilde{P}\left(v_{i}\right)$ to take this into account. Therefore, we obtain a new distribution $P\left(v_{i}\right) \equiv v_{i} \widetilde{P}\left(v_{i}\right)$, which equals $P\left(v_{i}\right)=v_{i}^{a / \log _{10} L}$. The corresponding time distribution to $P\left(v_{i}\right)$ is called $P\left(\tilde{t}_{i}\right)$ and satisfies

$$
P\left(\tilde{t}_{i}\right)=v_{i} \widetilde{P}\left(v_{i}\right) \frac{d v_{i}}{d \widetilde{t}_{i}}=v_{i}^{a / \log _{10} L} \frac{1}{\widetilde{t}_{i}^{2}}=\tilde{t}^{-2-a / \log _{10} L}
$$

Since we are treating the case for $\tilde{t} \approx \tilde{t}_{i}, P(\tilde{t})=P\left(\tilde{t}_{i}\right)$. Probability distribution $P(\tilde{t})$ is the distribution satisfied for very large $\tilde{t}$ in our problem, which means that $P(\tilde{t}) \sim \tilde{t}^{-\gamma^{(2)}}$, or

$$
\gamma^{(2)}=2+a / \log _{10} L
$$

In Fig. 12, we find the value of $\gamma^{(2)}$ as a function of $1 / \log _{10} L$, measured from simulations, for several values of the ratio $\lambda$. Data regression for these results yields the expression

$$
\gamma^{(2)}=2.08+0.81 \frac{1}{\log _{10} L} .
$$

The agreement between the predicted and the empirical results suggests that we have reached a regime where the traveling time values are dominated by the times on these bonds. This regime starts for times $\tau_{P}$ at constant pressure and $\tau_{C}^{(2)}$ at constant current. These times, in turn, scale with $\lambda$ with exponents $d_{P}=2.78 \pm 0.15$ and $d_{C}^{(2)}=2.90 \pm 0.09$, which are close to each other and to the quantities $d_{\times}$and $d_{B}+\tilde{\mu}$. This scaling is consistent with the hypothesis that the long-time regime appears when most of the fluid inside the cluster has been displaced and only the slowest parts of the cluster still preserve some original fluid. Since there are only two stagnation points for the homogeneous case of Sec. III, $P\left(t^{\prime}\right)$ is characterized by an exponential decay. This is in contrast to the $p=p_{c}$ case where a multifractal spectrum of stagnation points is present, generating a power-law for $P(\tilde{t})$. However, the long-time regime of the homogeneous case emerges in a time that scales as $\lambda^{d_{l}}$, with $d_{l}=3$, and since at $p=1, d_{B}$ $=2$, and $\tilde{\mu}=1$, this becomes consistent with our picture.

It is important to point out that the power-law behavior we observe implies that the $k$ moments $\left\langle\tilde{t}_{C}^{k}\right\rangle$ of the distribution $P\left(t_{C}\right)$ [and the equivalent for $P\left(t_{P}\right)$ ] diverge for all sufficiently large $k$. This appears to be in contrast with earlier work [15], where the high current limit $Q \rightarrow \infty$ has finite moments for all $k$ in a finite system. However, this apparent discrepancy is in fact due to the different conditions that are being considered. In Ref. [15], both convective and diffusive effects are present, and all tracers on a system are able to travel and eventually leave, even from very slow bonds, making the effect of the stagnation points negligible, and generating an exponential decay for the traveling time probability distribution. On the other hand, if no diffusion is present, as it is the case here, the presence of the multifractal distribution of the velocities in the bonds generates a powerlaw tail that makes the moments divergent; the tracers cannot diffuse away from the stagnation points.

\section{E. The full scaling forms of $P\left(t_{P}\right)$ and $P\left(t_{C}\right)$}

The results obtained in Secs. V and VI allow us to write a full set of scaling ansatz for the traveling time probability distributions under both constant current and constant pressure conditions. The ansatz takes into account the regions present in each of the distributions, as well as the short-time cutoff (before $t^{\prime}=t_{b r}^{\prime}$ ). For the cutoff, we follow closely the arguments presented in Ref. [13], where this behavior is accounted for by the use of a stretched exponential function.

For constant pressure, we write

$$
P\left(t_{P}\right) \sim t_{P}^{-g_{P}^{(1)}} F_{P}\left(t_{P}\right) G\left(\frac{t_{P}}{\lambda^{d_{P}}}\right),
$$

where functions $F_{P}(x)$ and $G(x)$ have the behaviors

$$
F_{P}(x) \sim \exp \left(-b x^{-\phi}\right)
$$


and

$$
G(x) \sim \begin{cases}\text { const, } & x \ll 1 \\ x^{-g_{P}^{(2)}+g_{P}^{(1)}}, & x \gg 1,\end{cases}
$$

and constants $b$ and $\phi$ are fitted by the data. For $\phi$, the data extracted from Fig. 2(a) yield $\phi=-1.42 \pm 0.03$. Exponents $g_{P}^{(1)}, g_{P}^{(2)}$, and $d_{P}$ are given by Eqs. (24), (26), and (28), respectively. In the unscaled coordinates, $P(t, r, L)$ under constant pressure is

$$
P(t, r, L) \sim\left(\frac{t}{r^{d_{B}+\tilde{\mu}}}\right)^{-g_{P}^{(1)}} F_{P}\left(\frac{t}{r^{d_{B}+\tilde{\mu}}}\right) G\left(\frac{t}{L^{d_{P}} r^{-d_{P}+d_{B}+\tilde{\mu}}}\right) .
$$

For the case of constant current, we have

$$
P\left(t_{C}\right) \sim t_{C}^{-g_{C}^{(1)}} F_{C}\left(t_{C}\right) H_{1}\left(\frac{t_{C}}{\lambda^{d_{C}^{(1)}}}\right) H_{2}\left(\frac{t_{C}}{\lambda^{d_{C}^{(2)}}}\right) .
$$

Function $F_{C}(x)$ corresponds to a stretched exponential

$$
F_{C}(x) \sim \exp \left(-c x^{-\theta}\right)
$$

where again constants $c$ and $\theta$ are fitted by the data. By completing the necessary transformations on Fig. 2(b), we obtain $\theta=-1.49 \pm 0.01$. Functions $H_{1}$ and $H_{2}$ are given by

$$
H_{1}(x) \sim \begin{cases}\text { const, } & x \ll 1 \\ x^{-g_{C}^{(2)}+g_{C}^{(1)},} & x \gg 1\end{cases}
$$

and

$$
H_{2}(x) \sim \begin{cases}\text { const, } & x \ll 1 \\ x^{-g_{C}^{(3)}+g_{C}^{(3)},} & x \gg 1\end{cases}
$$

Once again, exponents $g_{C}^{(1)}, g_{C}^{(2)}, g_{C}^{(3)}, d_{C}^{(1)}$, and $d_{C}^{(2)}$ are given by the Eqs. (33), (35), (38), (40), and (42), respectively. For the unscaled coordinates, we find

$$
\begin{aligned}
P(t, r, L) \sim & \left(\frac{t}{r^{d_{B}}}\right)^{-g_{C}^{(1)}} F_{C}\left(\frac{t}{r^{d_{B}}}\right) H_{1}\left(\frac{t}{L_{C}^{d_{C}^{(1)}} r^{-d_{C}^{(1)}+d_{B}}}\right) \\
& \times H_{2}\left(\frac{t}{L^{d_{C}^{(2)}} r^{-d_{C}^{(2)}+d_{B}}}\right) .
\end{aligned}
$$

An additional constraint that Eq. (51) has to satisfy is that of the equal-time theorem (we treat the scaled coordinates example). The flow of tracers occurs only on the backbone of the percolation clusters, which has volume proportional to $M_{B}$, and the total tracer current $Q$ is unity. Since we use all backbones that connect points $A$ and $B$, without requiring that they percolate throughout the entire system $L \times L$, we expect that the average time $\left\langle t_{C}\right\rangle$ scales with $\lambda$ exactly in the same way as $\left\langle M_{B}\right\rangle$ scales for a given $\lambda$.

The problem of the distribution of backbone masses $M_{B}$ of a percolation cluster defined between two points $A$ and $B$, separated by a distance $r$, for a given system of size $L \times L$, is treated in Ref. [48], and it is predicted that

$$
\left\langle M_{B}\right\rangle \sim \frac{L^{d_{B}}}{\lambda^{\psi}}, \quad \psi=0.37 \pm 0.02
$$

Therefore, applying the equal-time theorem,

$$
\left\langle t_{C}\right\rangle=\frac{\langle t\rangle}{r^{d_{B}}} \sim \frac{\left\langle M_{B}\right\rangle}{r^{d_{B}}} \sim \lambda^{d_{B}-\psi} .
$$

Calculating $\left\langle t_{C}\right\rangle$ from Eq. (51), we find that it scales as a power of $\lambda^{\xi}$, where $\xi$ depends on the exponents involved in Eq. (51). Noting that $g_{C}^{(2)}$ has a decreasing value that is $\lambda$ dependent and that this regime extends to $\tau_{C}^{(2)} \sim \lambda^{d \times}$, to satisfy Eq. (57), $\xi$ must satisfy

$$
\xi=d_{B}-\psi=\left(2-g_{C}^{(2)}\right) d_{\times}
$$

which implies that $g_{C}^{(2)} \approx 1.55$, very close to the value of exponent $g_{C}^{(1)}$. Therefore, we expect that

$$
g_{C}^{(2)} \rightarrow g_{C}^{(1)} \quad[\lambda \rightarrow \infty]
$$

and the power-law regions $\mathrm{III}_{C}$ and $\mathrm{IV}_{C}$ become one region, with exponent $g_{C}^{(1)}$ extending from the maximum of $P\left(t_{C}\right)$ to the crossover time $\tau_{C}^{(2)}$.

Finally, regarding the validity of our results in true fieldsize porous media, we hypothesize that the form of the scaling ansatz presented still holds, even if the values of the exponents change. This issue must be resolved by additional studies.

\section{CONCLUSIONS}

We establish that the distributions of traveling times obey the general scaling relations $P(t, r, L)=\left(1 / r^{z}\right) P\left(t / r^{z}, 1, \lambda\right)$, and the production curve satisfies $C(t, r, L)=C\left(t / r^{z}, 1, \lambda\right)$. For constant pressure conditions, $z=d_{B}+\tilde{\mu}$, and for constant current, $z=d_{B}$. This relates the scaling of the traveling time to the scaling of two basic cluster properties: backbone and conductivity.

Using the rescaled times $t_{P} \equiv t / r^{d_{B}+\tilde{\mu}}$ and $t_{C} \equiv t / r^{d_{B}}$, we have determined the dependence of $P\left(t_{P}\right)=P\left(t_{P}, 1, \lambda\right)$ and $P\left(t_{C}\right)=P\left(t_{C}, 1, \lambda\right)$ on the geometric parameter $\lambda$ and have observed several power-law regions. We obtain the exponents for the power-law regions and crossover times of $P\left(t_{P}\right)$ and $P\left(t_{C}\right)$ by convolution of functions $P\left(t_{P} \mid R\right)$ and $\Phi(R)$, expressed as functions of exponents $g_{R}, d_{R}, d_{\times}$, $\gamma^{(1)}$, and $\gamma^{(2)}$. The crossover times themselves scale as powers of ratio $\lambda$. We propose relations between $d_{R}$ and $d_{\times}$and the fundamental percolation exponents $d_{B}$ and $\tilde{\mu}$. Using arguments based on multifractality, we also propose the relation $\gamma^{(2)}=2+a / \log _{10} L$. The exponent $\gamma^{(1)}$ is not yet explained (see Table II). The full scaling forms of $P\left(t_{P}\right)$ and $P\left(t_{C}\right)$ are expressed in two scaling ansatz [Eqs. (47) and (51)] that contain all their observed regions and crossover times. 
For the longest times, the crossover occurs for the $p=1$ case at a time that scales as $\lambda^{d_{l}}$, with $d_{l}=3$, which can be interpreted as $d_{B}+\tilde{\mu}$ under homogeneous conditions. For the $p=p_{c}$ case, the crossover to the long-time region scales as $\lambda^{d} \times$, regardless of the pumping conditions (constant current or constant pressure). We hypothesize that $d_{\times}$and $d_{B}+\tilde{\mu}$ are the same exponent and propose that the transition to the long time regime occurs similarly at different values of the occupation probability $p$.

\section{ACKNOWLEDGMENT}

We thank Intevep for financial support.

\section{APPENDIX: ANALYTICAL SOLUTION FOR THE HOMOGENEOUS CASE}

The equations for the stationary ideal flow [43] are $\nabla^{2} P$ $=0$ and $\vec{v}=\vec{\nabla} P$, where $P$ is the pressure, $\vec{v}$ is the velocity, and $\nabla^{2}$ is the Laplace operator. In two dimensions, the solution is given by an arbitrary analytical function $f(z)$ of complex variable $z=x+i y, \quad$ and $P(x, y)=\operatorname{Re} f(z), \quad v_{x}$ $=\operatorname{Re} f^{\prime}(z)$, and $v_{y}=-\operatorname{Im} f^{\prime}(z)$. The equation of a streamline is given by $\phi=\operatorname{Im} f(z)$, where $\phi$ is a parameter. The flow between two wells $A$ and $B$, located at points $( \pm r / 2,0)$ in the circular reservoir $x^{2}+y^{2} \leqslant L^{2} / 4$, is given by

$$
f(z)=\ln \left(z+\frac{r}{2}\right)-\ln \left(z-\frac{r}{2}\right)+\ln \left(z+\frac{L^{2}}{2 r}\right)-\ln \left(z-\frac{L^{2}}{2 r}\right) .
$$

The circular boundary of the reservoir satisfies the equation $\phi=\pi$. The fastest streamline connecting $A$ and $B$ corresponds to $\phi=0$ and an arbitrary streamline forms angles $\phi$ and $(\pi-\phi)$ with the $x$ axis at points $A$ and $B$. The traveling time along a streamline can be found as a contour integral

$$
t=\int_{A}^{B} \frac{d z}{\overline{f^{\prime}(z)}}=\int_{A}^{B} \frac{d x}{v_{x}}=\int_{A}^{B} \frac{d y}{v_{y}},
$$

where $\overline{f^{\prime}(z)}$ is the complex conjugate of $f^{\prime}(z)$. The traveling time $t(\phi)$ can also be found by differentiation of the area $S(\phi)$ between two streamlines corresponding to different values of $\phi$,

$$
t(\phi)=\frac{d S(\phi)}{d \phi}
$$

which is another manifestation of the equal-time theorem. The concentration of oil arriving at well $B$ at time $t$, i.e., the production curve $C(t, r)$, is given by the inverse function $\phi(t)$

$$
C(t, r)=\frac{\pi-\phi(t)}{\pi}
$$

In the unbounded case $L \rightarrow \infty$, the streamlines are circles and $t(\phi)$ is given by an elementary formula

$$
t=\frac{r^{2}}{2 \sin ^{2} \phi}\left(1-\frac{\phi \cos \phi}{\sin \phi}\right)
$$

Thus $t$ has dimensionality of area and, therefore, in the following, we use the scaled time $t^{\prime}=t / r^{2}$. Accordingly, $C(t, r)$ has the scaling property

$$
C(t, r)=C\left(t^{\prime}=t / r^{2}, 1\right) .
$$

In the interest of briefness, we use $C\left(t^{\prime}\right) \equiv C\left(t^{\prime}, 1\right)$. For small $\phi \rightarrow 0, t^{\prime}$ is given by the expansion

$$
t^{\prime}=\frac{1}{6}+\frac{1}{15} \phi^{2}+O\left(\phi^{4}\right) .
$$

For large $\phi \rightarrow \pi, t^{\prime} \rightarrow \infty$ and

$$
t^{\prime}=\frac{\pi}{2(\pi-\phi)^{3}}\left[1+O\left((\pi-\phi)^{2}\right)\right]
$$

The breakthrough time $t_{b r}^{\prime}$ corresponds to the fastest streamline $\phi=0$ and thus is given by

$$
t_{b r}^{\prime}=\lim _{\phi \rightarrow 0} t^{\prime}(\phi)=\frac{1}{6} \text {. }
$$

Immediately after breakthrough, the concentration of oil drops as

$$
C\left(t^{\prime}\right)=1-K \sqrt{t^{\prime}-t_{b r}^{\prime}}+O\left(\left(t^{\prime}-t_{b r}^{\prime}\right)^{3 / 2}\right),
$$

where $K=\sqrt{15} / \pi$. When $t^{\prime} \rightarrow \infty$, the concentration decays as a power-law,

$$
C\left(t^{\prime}\right)=\left(\frac{1}{2 \pi^{2} t^{\prime}}\right)^{1 / 3}+O\left(\frac{1}{t^{\prime}}\right)
$$

The crossover time from the initial fast decay to the slow power-law decay is approximately equal to time $t_{1 / 2}^{\prime}$, defined as the time when the concentration drops by a factor of 2 , or $t_{1 / 2}^{\prime}=t^{\prime}(\pi / 2)=1 / 2$.

In the bounded case, the production curve for scaled time depends only on the ratio $\lambda \equiv L / r$, and similar to the result of Eq. (A6), we find that

$$
C(t, r, L)=C\left(t^{\prime}=t / r^{2}, 1, \lambda\right) .
$$

Production curve $C\left(t^{\prime}, \lambda\right) \equiv C\left(t^{\prime}=t / r^{2}, 1, \lambda\right)$ can be expressed via elliptical functions. Integrating Eq. (A2) along the fastest streamline gives

$$
t_{b r}^{\prime}=\frac{1+\lambda^{6}+\left(1-\lambda^{2}\right)^{3}}{12\left(1+\lambda^{2}\right)}+\frac{\lambda\left(1-\lambda^{2}\right)^{2}}{8\left(1+\lambda^{2}\right)} \ln \left(\frac{\lambda-1}{\lambda+1}\right),
$$

which in the limit $\lambda \rightarrow \infty$ yields

$$
t_{b r}^{\prime}=\frac{1}{6}-\frac{2}{15} \lambda^{-2}+o\left(\lambda^{-2}\right) .
$$


Constant $K$ in the initial fast decay can be obtained by expanding the equations of short streamlines in powers of $\phi$, resulting in lengthy elementary functions of $\lambda$. Expanding the latter in powers of $\lambda^{-1}$ yields

$$
K=\frac{\sqrt{15}}{\pi}\left[1+\frac{10}{7} \lambda^{-2}+o\left(\lambda^{-2}\right)\right] .
$$

The half-time is given by integration along the $\phi=\pi / 2$ streamline,

$$
\begin{aligned}
t_{1 / 2}^{\prime} & =\frac{1}{4}\left\{1+\lambda^{2}-\frac{\left(1+\lambda^{2}\right)^{3}}{4 \lambda} \arcsin \left[\frac{4}{\left(1+\lambda^{2}\right) \sqrt{\lambda^{2}+6+\lambda^{-2}}}\right]\right\} \\
& =\frac{1}{2}+O\left(\lambda^{-2}\right) .
\end{aligned}
$$

Finally, as $t^{\prime} \rightarrow \infty$ and $\phi \rightarrow \pi$, the entire reservoir is swept out except in the vicinity of the two stagnation points $( \pm L / 2,0)$, where the velocity is equal to zero. Integrating Eq. (A2) along the reservoir boundary and the segments connecting the wells and stagnation points, with the exception of a small vicinity of the stagnation point of order $\sqrt{\pi-\phi}$, gives

$$
t^{\prime}=t_{o}^{\prime}-t_{\ell}^{\prime} \ln (\pi-\phi)+o(1),
$$

where $t_{o}^{\prime}$ and $t_{\ell}^{\prime}$ are functions of $\lambda$,

$$
t_{\ell}^{\prime}=\frac{1}{8} \frac{\lambda\left(\lambda^{2}-1\right)^{2}}{\left(\lambda^{2}+1\right)}=\frac{\lambda^{3}}{8}+O(\lambda)
$$

and

$$
\begin{aligned}
t_{o}^{\prime}= & t_{\ell}^{\prime}\left[\frac{2\left(2-3 \lambda-3 \lambda^{2}+10 \lambda^{3}+3 \lambda^{4}-3 \lambda^{5}\right)}{3 \lambda\left(\lambda^{2}-1\right)^{2}}\right. \\
& \left.+\ln \frac{16 \lambda\left(\lambda^{2}+1\right)}{\left(\lambda^{2}-1\right)(\lambda+1)^{2}}\right] .
\end{aligned}
$$

This yields the exponential decay of $C\left(t^{\prime}\right)$ at $t^{\prime} \rightarrow \infty$

$$
C\left(t^{\prime}\right)=\frac{1}{\pi} \exp \left[-\frac{\left(t^{\prime}-t_{o}^{\prime}\right)}{t_{\ell}^{\prime}}\right][1+o(1)],
$$

where $t_{\ell}^{\prime}$ plays the role of the characteristic time. If $t_{\ell}^{\prime}>t_{1 / 2}^{\prime}$ $(\lambda \gg 1)$, an intermediate power-law decay is present between $t_{1 / 2}^{\prime}$ and $t_{\ell}^{\prime}$, with the scaling form of Eq. (A11). In this case, $t_{\ell}^{\prime}$ also plays the role of the crossover time from the powerlaw to the exponential decay.
[1] P.R. King, in North Sea Oil and Gas Reservoirs III, edited by A.T. Buller et al. (Graham and Trotman, London, 1990).

[2] D. Stauffer and A. Aharony, Introduction to Percolation Theory, 2nd ed. (Taylor \& Francis, London, 1994).

[3] M. Sahimi, Flow and Transport in Porous Media and Fractured Rock (VCH, Boston, 1995), and references therein.

[4] M. Sahimi, Applications of Percolation Theory (Taylor \& Francis, London, 1994).

[5] Fractals and Disordered Systems, 2nd ed., edited by A. Bunde and S. Havlin (Springer, Berlin, 1996), and references therein.

[6] D. Ben-Avraham and S. Havlin, Diffusion and Reactions in Fractals and Disordered Systems (Cambridge University Press, Cambridge, 2000).

[7] J.M. Ziman, Models of Disorder (Cambridge University Press, Cambridge, 1979).

[8] The Fractal Approach to Heterogeneous Chemistry: Surfaces, Colloids, Polymers, edited by D. Avnir (Wiley, Chichester, 1989).

[9] S. Havlin and D. Ben-Avraham, Adv. Phys. 36, 695 (1987).

[10] P.G. Saffman, J. Fluid Mech. 6, 321 (1959).

[11] N.V. Dokholyan, Y. Lee, S.V. Buldyrev, S. Havlin, P.R. King, and H.E. Stanley, J. Stat. Phys. 93, 603 (1998).

[12] Y. Lee, J.S. Andrade, S.V. Buldyrev, N.V. Dokholyan, S. Havlin, P.R. King, G. Paul, and H.E. Stanley, Phys. Rev. E 60, 3425 (1999).

[13] J.S. Andrade, S.V. Buldyrev, N.V. Dokholyan, S. Havlin, P.R. King, Y. Lee, G. Paul, and H.E. Stanley, Phys. Rev. E 62, 8270 (2000).

[14] J.-C. Bacri et al., in Hydrodynamics of Dispersed Media, ed- ited by J.P. Hulin, A.M. Cazabat, E. Guyon, and F. Carmona (Elsevier, Amsterdam, 1990), p. 249

[15] J. Koplik, S. Redner, and D. Wilkinson, Phys. Rev. A 37, 2619 (1988).

[16] V. Ambegaokar, B.I. Halperin, and J.S. Langer, Phys. Rev. B 4, 2612 (1971).

[17] M. Sahimi, Rev. Mod. Phys. 65, 1393 (1993).

[18] E.T. Gawlinski and H.E. Stanley, J. Phys. A 14, L291 (1981).

[19] A. Geiger and H.E. Stanley, Phys. Rev. Lett. 49, 1895 (1982).

[20] A.J. Katz and A.H. Thompson, Phys. Rev. B 34, 8179 (1986); J. Geophys. B92, 599 (1987).

[21] Hernan A. Makse, Glenn W. Davies, Shlomo Havlin, Plamen Ch. Ivanov, Peter R. King, and H.E. Stanley, Phys. Rev. E 54, 3129 (1996).

[22] T.A. Hewett (unpublished); T.A. Hewett and R.A. Behrens, SPE Form. Eval. 5, 217 (1990).

[23] A.D. Araújo, A.A. Moreira, H.A. Makse, H.E. Stanley, and J.S. Andrade, Phys. Rev. E 66, 046304 (2002).

[24] M. Sahimi, J. Phys. I 4, 1263 (1994); M. Sahimi and M.A. Knackstedt, ibid. 4, 1269 (1994); M. Sahimi, AIChE J. 41, 229 (1995).

[25] S. Prakash, S. Havlin, M. Schwartz, and H.E. Stanley, Phys. Rev. A 46, R1724 (1992).

[26] Z. Alexandrowicz, Phys. Lett. 80A, 284 (1980).

[27] R. Pike and H.E. Stanley, J. Phys. A 14, L169 (1981).

[28] H.J. Herrmann and H.E. Stanley, J. Phys. A 21, L829 (1988).

[29] P. Grassberger, J. Phys. A 25, 5475 (1992).

[30] P. Ray, J. Phys. A 18, L657 (1985).

[31] M. Barma and P. Ray, Phys. Rev. B 34, 3403 (1986). 
[32] A.U. Neumann and S. Havlin, J. Stat. Phys. 52, 203 (1988).

[33] R.M. Ziff, J. Phys. A 32, L457 (1999).

[34] J.R. de Dreuzy, P. Davy, and B. Berkowitz, Phys. Rev. E 64, 056305 (2001).

[35] See, e.g., J.G. Zabolitzky, Phys. Rev. B 30, 4077 (1984); H.J. Herrmann, B. Derrida, and J. Vannimenus, ibid. 30, 4080 (1984); D.C. Hong, S. Havlin, H.J. Herrmann, and H.E. Stanley, ibid. 30, 4083 (1984); C.J. Lobb and D.J. Frank, ibid. 30, 4090 (1984); L. de Arcangelis, S. Redner, and A. Coniglio, ibid. 31, 4725 (1985).

[36] M. Barthélémy, S.V. Buldyrev, S. Havlin, and H.E. Stanley, Phys. Rev. E 61, R3283 (2000).

[37] M. Murat and A. Aharony, Phys. Rev. Lett. 57, 1875 (1986).

[38] J.S. Andrade, A.D. Araujo, S.V. Buldyrev, S. Havlin, and H.E. Stanley, Phys. Rev. E 63, 051403 (2001).

[39] L. Paterson, Phys. Rev. Lett. 52, 1621 (1984).

[40] J.D. Sherwood and J. Nittmann, J. Phys. (Paris) 47, 15 (1986)
[41] P.R. King, J. Phys. A 20, L529 (1987).

[42] S. Redner, A Guide to First-Passage Processes (Cambridge University Press, Cambridge, 2001).

[43] J. Koplik, S. Redner, and E.J. Hinch, Phys. Rev. E 50, 4650 (1994).

[44] M. Sahimi, H.T. Davis, and L.E. Scriven, Chem. Eng. Commun. 23, 329 (1983); M. Sahimi, A.L. Heiba, B.D. Hughes, L.E. Scriven, and H.T. Davis, Chem. Eng. Sci. 41, 2103 (1986); 41, 2123 (1986); M. Sahimi and A.O. Imdakm, J. Phys. A 21, 3833 (1988).

[45] P. Grassberger, Physica A 262, 251 (1999).

[46] G. Paul, S.V. Buldyrev, N.V. Dokholyan, S. Havlin, P.R. King, Y. Lee, and H.E. Stanley, Phys. Rev. E 61, 3435 (2000).

[47] G.G. Batrouni, A. Hansen, and S. Roux, Phys. Rev. A 38, 3820 (1988).

[48] M. Barthélémy, S.V. Buldyrev, S. Havlin, and H.E. Stanley, Phys. Rev. E 60, R1123 (1999). 